\title{
Autökologische Analyse der in schweizerischen Fliessgewässern häufig vorkommenden Ciliatenarten und ihre Eignung als Bioindikatoren
}

\author{
Von F. Stössel \\ Eidg. Anstalt für Wasserversorgung, Abwasserreinigung und Gewässerschutz. (EAWAG). \\ CH-8600 Dübendorf.
}

Manuskript eingegangen am 16. Februar 1979

\begin{abstract}
An autecological study of ciliated protozoa in Swiss streams and their meaning as bioindicators

Microscopic analyses were made of 'Aufwuchs' samples taken at a total of 439 sampling stations in 194 Swiss streams. Special emphasis was placed on the autecology of ciliated protozoa. Water samples were collected at each sampling station and submitted to physical and chemical analysis. The occurrence and optimal range of 20 ciliate species is described as a function of eight chemical and two physical parameters ( 33 species for disssolved organic carbon).
\end{abstract}

\section{INHALTSVERZEICHNIS}

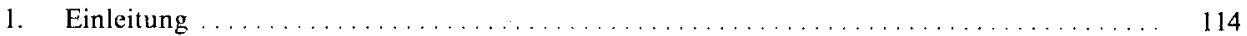

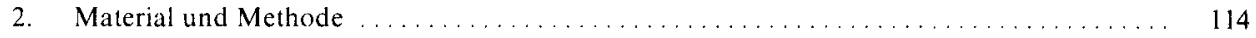

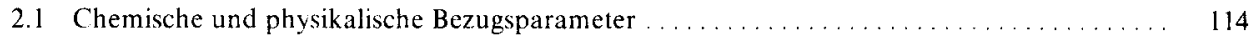

2.2 Verteilung und Klassenbildung der Bezugsparameter $\ldots \ldots \ldots \ldots \ldots \ldots \ldots \ldots \ldots \ldots \ldots \ldots$

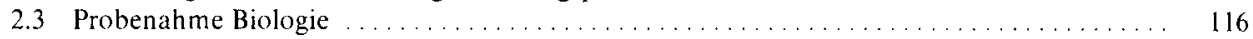

2.4 Berechnung des Artenvorkommens . . . . . . . . . . . . . . . . . . . . . . 119

2.5 Fehlergrenzen der prozentualen Belegung $\ldots \ldots \ldots \ldots \ldots \ldots \ldots \ldots \ldots \ldots \ldots \ldots \ldots$

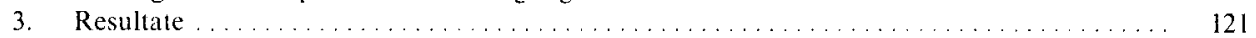

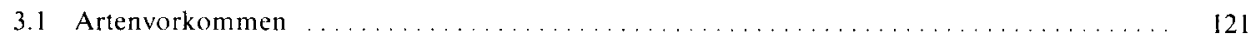

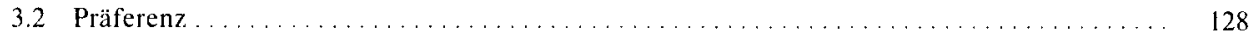

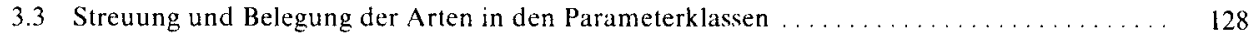

4. Der Einfluss weiterer, nicht anthropogen bedingter Parameter auf das Vorkommen von

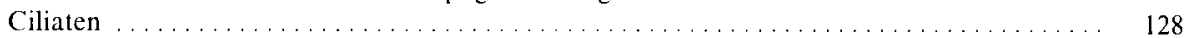

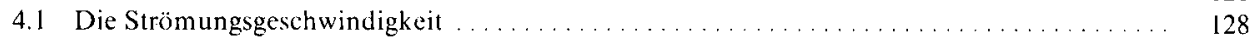

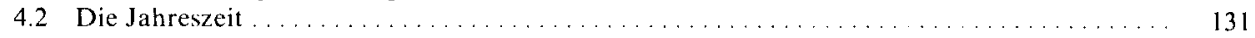

5. Vergleich mit autökologischen Angaben anderer Autoren $\ldots \ldots \ldots \ldots \ldots \ldots \ldots \ldots \ldots$

6. Die Eignung der Ciliaten als Bioindikatoren $\ldots \ldots \ldots \ldots \ldots \ldots \ldots \ldots \ldots \ldots \ldots \ldots \ldots \ldots$

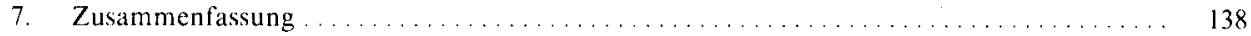

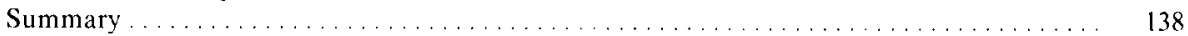

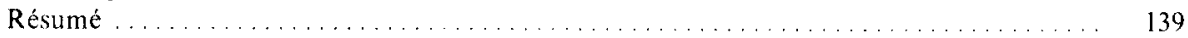

Verdankungen $\ldots \ldots \ldots \ldots \ldots \ldots \ldots \ldots \ldots \ldots \ldots \ldots \ldots \ldots \ldots \ldots \ldots \ldots \ldots$

Literaturverzeichnis . . . . . . . . . . . . . . . . . . . . . . . . . . . . . 139 


\section{Einleitung}

Der Organismenbestand eines fliessenden Gewässers liefert im allgemeinen eine rasche, allerdings nur grobe Information über den Belastungszustand an der Untersuchungsstelle. Dies dürfte einer der Gründe sein, weshalb 70 Jahre nach der Einführung der «tierischen Saprobien» durch Kolkwitz und Marsson [10] immer noch neue Bioindizes. entstehen. mit welchen anthropogene Einflüsse in Gewässern taxiert werden sollen. Ein anderer Grund liegt in der Tatsache, dass das Saprobiensystem nicht universell anwendbar ist. Die Kritik Steinmanns [22] am System, wonach die Autökologie und Synökologie der Leitformen (1918) weitgehend unbekannt sei, gilt zu einem gewissen Teil auch noch heute. In der Literatur über Ciliaten finden sich ökologische Angaben meist nur in Form von Toleranzgrenzen des Vorkommens; die Verteilung der Arten zwischen den Limiten ist hingegen nur vereinzelt angegeben [3, 5, 8, 13]. Somit besteht auf dem Gebiet der Autökologie und namentlich ihrer Abstützung mit analytischen Gewässerdaten noch immer eine grosse Informationslücke.

Die hier vorliegende Arbeit ist ein Beitrag zur bioindikatorischen Bedeutung von Ciliaten. Das Grundlagenmaterial für die autökologischen Abklärungen stammt aus der unter dem Projektnamen «Mapos» 1974 und 1975 durchgeführten Erhebung [15]. Der grösste Teil der Ciliaten gehört ökologisch zu den Bakterienfressern und ist somit von der durchschnittlichen Belastung eines Gewässers mit bakteriell abbaubarem Material abhängig. Diese Verknüpfung zwischen organischer Belastung und Ciliatendichte zeigt sich besonders auffällig im Aufwuchs des Fliesswasserbenthos. Dank ihrer kurzen Reproduktionszeit vermögen sich die Ciliaten veränderten Nahrungsbedingungen innert kurzer Zeit anzupassen. Diese quantitative Veränderung der Ciliatenfauna wird bei erhöhten Belastungen makroskopisch sichtbar, indem sich durch sessile Ciliaten der Ordnung Peritricha an Steinen des Benthos Kolonien in Form von weisslichen Flecken bilden. Wie verhalten sich aber die einzelnen Arten im Spektrum verschiedener chemischer und physikalischer Parameter?

\section{Material und Methode}

\subsection{Chemische und physikalische Bezugsparameter}

Zur chemischen Analyse des Wassers wurden an jeder Probenahmestelle zwei. höchstens jedoch drei Stichproben erhoben. Mit unendlich vielen Stichproben liesse sich eine Fliesswasserstelle durch die verschiedenen physikalischen und chemischen Parameter ideal beschreiben. Eine solche ideale Probenahme ist jedoch höchstens angenähert zu erreichen. Zobrist, Davis und Hegi [26] haben einen kleinen Mittellandfluss (mittlere Abflussmenge $8-10 \mathrm{~m}^{3} / \mathrm{s}$ ) mit wöchentlichen Stichproben untersucht. Die Konzentrationswerte der untersuchten Parameter aus gesamthaft 95 Stichproben zeigen Häufigkeitsverteilungen, die entweder dem Typus «normal» oder «log-normal» entsprechen. In einer weiteren Arbeit derselben Autoren [27] ergaben sich für andere Fliessgewässer der Schweiz dieselben Häufigkeitsverteilungen. Nun stellt sich die Frage. wieweit sich die Häufigkeitsverteilung eines Parame- 
ters durch Stichproben beschreiben lässt. In unserem Falle ist diese Beschreibung mit den wenigen Stichproben pro Stelle nicht möglich, doch liegen die meisten Werte mit grosser Wahrscheinlichkeit im Bereich der häufigsten Konzentrationen an der betreffenden Stelle. Diese sind wiederum von der zeitlichen Verteilung der Abflussmenge und vơn anthropogenen Einflüssen abhängig.

Nehmen wir an, wir hätten an jedem Probenort eine Idealprobe desselben Parameters erhoben, so lieferte die Summe aller dieser Idealproben die anzustrebende Aussagegenauigkeit und damit auch die Häufigkeitsverteilung des durchschnittlichen "schweizerischen Fliessgewässers». Bestimmen wir nun die Verteilung der Häufigkeiten sämtlicher Mittelwerte des Parameters, so erhalten wir ebenfalls eine Verteilung vom log-normalen Typus (siehe Kapitel 2.2). Dieses Verteilungsbild entspricht aufgrund der erwähnten Arbeiten [26, 27] unseren Erwartungen. Die zu einer Summenhäufigkeit verarbeiteten Konzentrationsmittelwerte sind nun nicht wie in der Probe einer einzelnen Stelle von Mensch und Abflussmenge allein beeinflusst, sondern zusätzlich von geologischen bzw. geochemischen Verhältnissen. Die Zusammenfassung zu einer einzigen Stichprobenmenge ist zulässig, da die arithmetischen Mittelwerte eines Parameters aller untersuchten Stellen log-normal verteilt sind. Die Zahl der verwendeten Werte dieser «Gesamtprobe Schweiz» steigt somit pro Parameter auf 439. Mit einer solchen Probenzahl lassen sich Mittelwerte, Medianwerte, die am häufigsten vorkommenden Konzentrationswerte und auch die Extremwerte der Häufigkeitsverteilung sehr wohl beschreiben. Allerdings ist klarzumachen, dass die Auswahl der Probenahmestellen nicht einem Zufallsmuster entsprach, sondern es wurden recht bewusste Kriterien angewendet. Dies sind vor allem: Abflussmenge $\geqslant 1 \mathrm{~m}^{3} / \mathrm{s}$, Einflüsse von Siedlungen, Mündungen in andere Gewässer. Ebenso wesentlich ist die Feststellung, dass mit unseren Daten der Chemismus der einzelnen Stellen der Stichprobenentnahmen nur unsicher charakterisiert werden kann. Hingegen ist unser Stichprobenmaterial geeignet, die ökologische Breite der einzelnen Art aufzuzeigen.

Anschliessend sind die gemessenen Parameter und die Analysenmethodik beschrieben:

Phosphor: Bestimmung des Gesamtphosphors aus dem Rohwasser und dem Filtrat nach Schmid und Ambühl [19]. Der partikuläre Phosphor wurde aus der Differenz der beiden Konzentrationen errechnet.

Stickstoff: Für unsere Auswertungen wurden der Nitratstickstoff nach Müller und Wiedemann [12] und der Kjeldahl-Stickstoff nach Schmid [18] bestimmt. Der Gesamtstickstoff wurde nur durch Summieren der beiden Konzentrationen errechnet, da der dazugehörige Nitritstickstoff infolge von Schwierigkeiten der Probenkonservierung im Felde entfiel.

Kohlenstoff: Als Bezugsparameter für den Kohlenstoff benützten wir den gelösten organischen Kohlenstoff (DOC), der nach der Methode von Axt [2] bestimmt wurde.

Kalzium: Bestimmung dieses geochemischen Parameters nach der Methode von Patton und Reeder [14].

Leitfähigkeit: Die Leitfähigkeit wurde an Ort und Stelle mit einem WTW-Gerät des Typs LF 56 gemessen. Zur Vereinheitlichung wurden die Messwerte auf eine Wassertemperatur von $20^{\circ} \mathrm{C}$ umgerechnet. 
Wassertemperatur: Messung mit einem Quecksilberthermometer auf $0,2^{\circ} \mathrm{C}$ genau. Sauerstoffgehalt und -sättigung: Der sonst in keiner Arbeit fehlende Sauerstoffgehalt und die Sauerstoffsättigung wurden ermittelt [24], für diese Arbeit jedoch nicht als Bezugsparameter einbezogen. Der Grund liegt in der ausserordentlich guten Sauerstoffversorgung unserer Fliessgewässer. 404 Werte lagen über $90 \%, 33$ Werte zwischen 70 und $90 \%$ und nur 2 Werte unter $70 \%$ Sauerstoffsättigung. Es muss allerdings betont werden, dass es sich hier um Tageswerte handelt, da keine Nachtproben entnommen wurden.

Fliessgeschwindigkeit und Durchflussvolumen: Die Messung der Fliessgeschwindigkeit erfolgte mit einem elektronischen Flügelmessgerät, Typ «mini-water», der Firma E. Schildknecht in Gossau (Zürich) auf zwei Dritteln Tiefe ab Grund und an mindestens drei Stellen im Querprofil.

Die Durchflussmenge wurde durch eine einfache Rechnung aus Tiefe, Fliessgeschwindigkeit und Entfernung der Messpunkte vom Ufer angenähert. Von den 439 untersuchten Stellen lagen etwa 220 an kleinen Fliessgewässern mit bis zu $2 \mathrm{~m}^{3} / \mathrm{s}, 130$ an Stellen mit $2-10 \mathrm{~m}^{3} / \mathrm{s}$ und der Rest an Stellen mit einem Abflussvolumen von 10 bis $400 \mathrm{~m}^{3 /} / \mathrm{s}$.

\subsection{Verteilung und Klassenbildung der Bezugsparameter}

Die Daten der abiotischen Faktoren wurden in 10 geometrisch abgestufte Klassen aufgeteilt. Eine arithmetische Klassenbildung kam aufgrund der Verteilung vom log-normalen Typus des vorliegenden Datenmaterials nicht in Frage, da zu viele obere Klassen leer oder zumindest sehr schwach besetzt gewesen wären. Die oberste Histogrammdarstellung der Abb. 2 zeigt ein Beispiel des in 10 arithmetische Klassen aufgeteilten gelösten organischen Kohlenstoffs, das eindeutig die obenerwähnte, stark schiefe Verteilung der Daten darlegt. Die geometrischen Schritte wurden folgendermassen bestimmt:

$$
\text { Klassenbreite }=\frac{\log \text { Maximalwert }-\log \text { Minimalwert }}{\text { Anzahl Klassen }}
$$

In den Abb. 1 a-k sind die Bezugsparameter als Histogrammdarstellungen wiedergegeben. Die Ordinate repräsentiert die Anzahl der biologisch und chemisch untersuchten Stellen ( = Häufigkeit der Werte), die Abszisse die Analysen-oder Messwerte des betreffenden Parameters, in Klassen aufgeteilt.

\subsection{Probenahme Biologie}

An jeder Probenahmestelle erhoben wir zwei getrennte Sammelproben des Aufwuchses. Zu diesem Zwecke wurden locker dem Grunde aufliegende grössere Steine auf der Ober- wie Unterseite abgekratzt. Um ein möglichst vollständiges Artenspektrum der an der Probenahmestelle vorkommenden Ciliaten zu erhalten, wurden Steine des gesamten Querschnitts auf einer Fliesswasserstrecke von bis zu etwa $200 \mathrm{~m}$ entnommen. Dies ermöglichte uns. Habitate mit verschiedenstem Lichteinfall 
GESAMTPHOSPHOR FILTRIERT GESAMTPHOSPHOR ROH
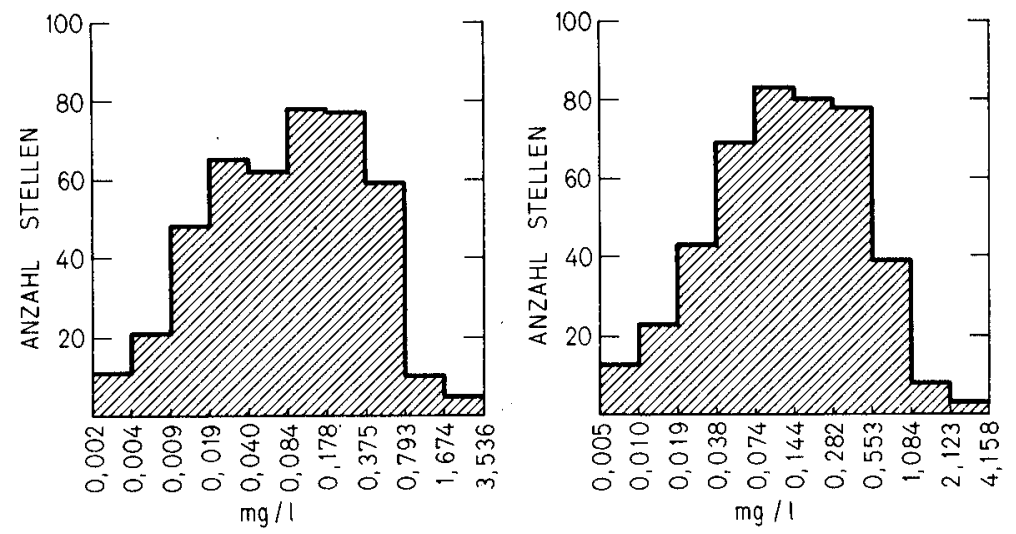

PHOSPHOR PARTIKULÄR

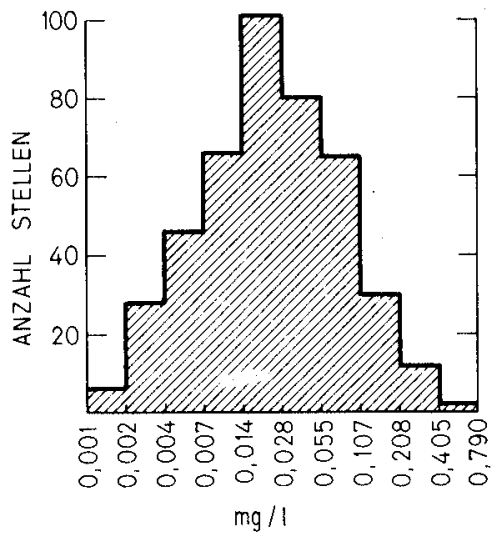

\section{GESAMTSTICKSTOFF}

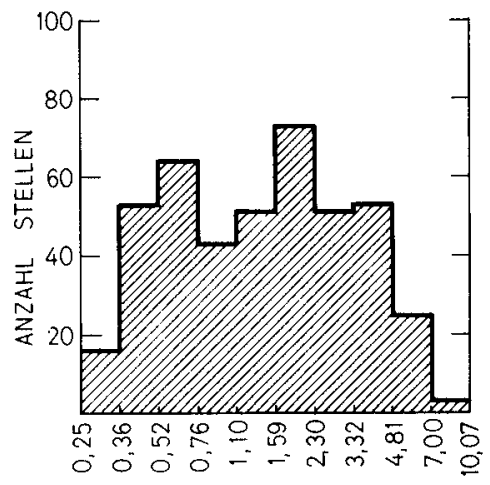

$\mathrm{mg} / \mathrm{l}$
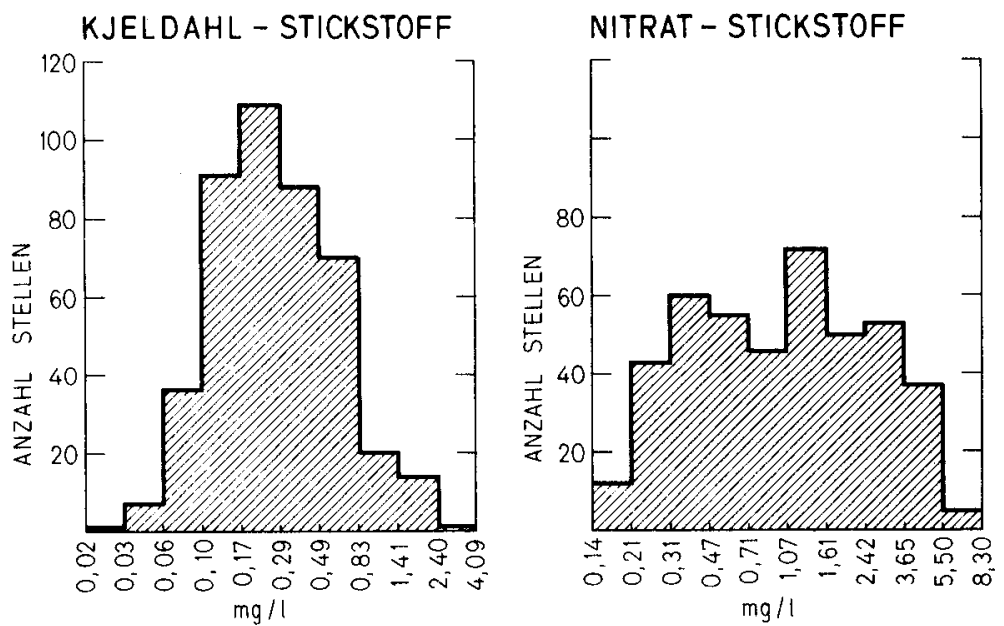

Abb. 1 a-f. Verteilung der Bezugsparameter.

Figure 1 a-f. Distribution of parameter values. 

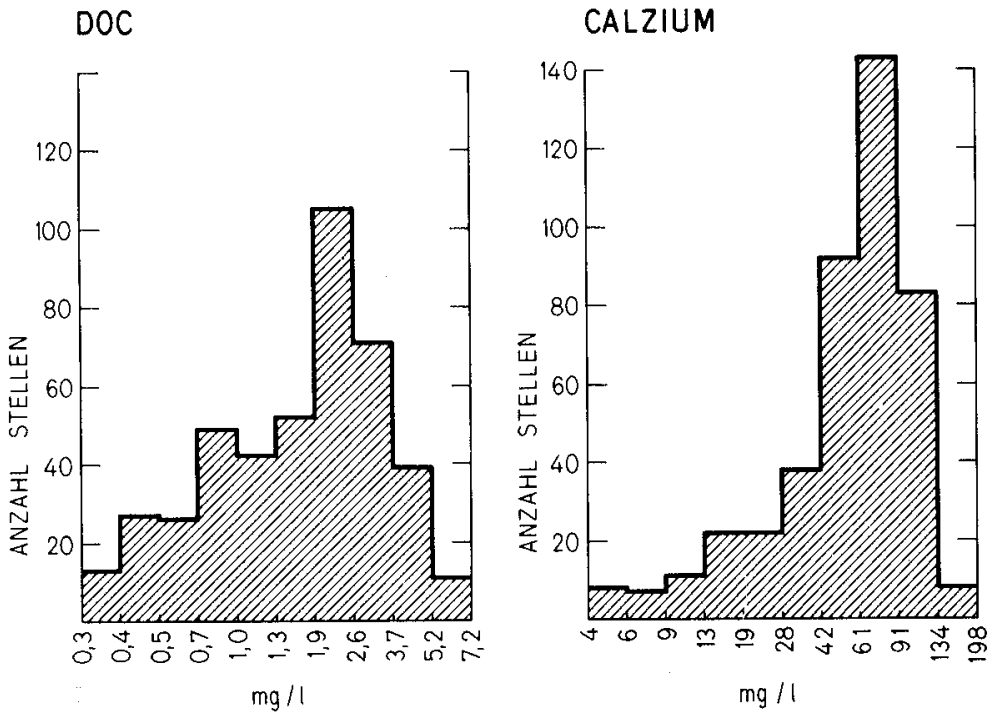

\section{LEITFÄHIGKEIT}

\section{WASSERTEMPERATUR}
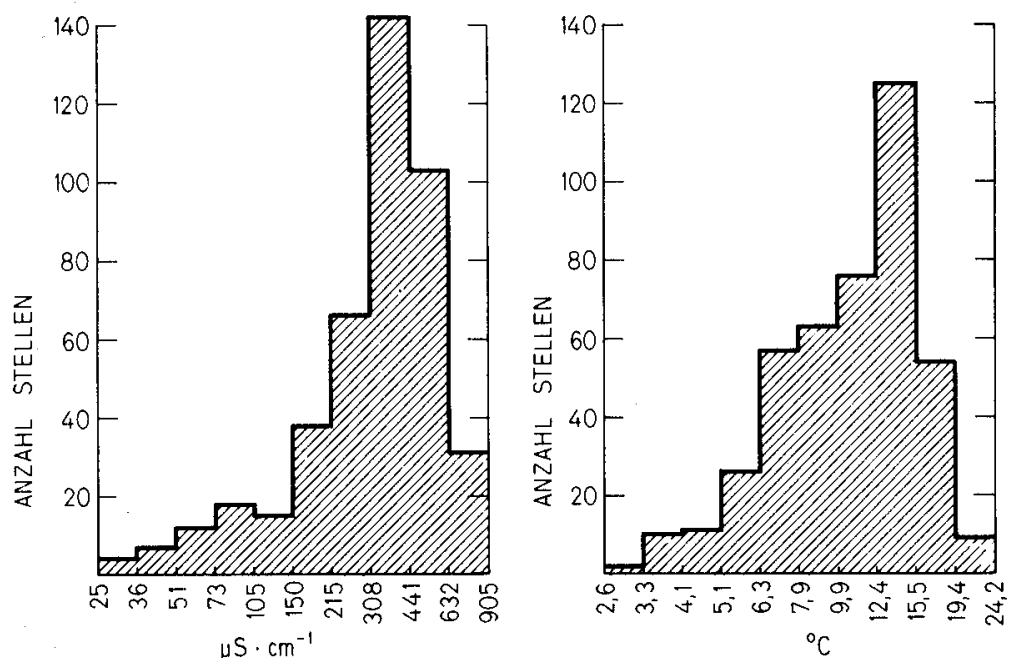

Abb. I g-k. Bei der Interpretation der Verteilung der Bezugsparameter ist der logarithmische Maßstab der Klassen zu beachten.

Figure $1 \mathrm{~g}-\mathrm{k}$. Distribution of values of chemical and physical parameters in water samples. Note logarithmic scale. 
und unterschiedlichen Fliessgeschwindigkeiten zu erfassen. In tieferen Gewässern erfolgte die Probenahme durch einen Taucher, der die Steine des Benthos heraufholte.

Die mikroskopische Untersuchung der lebenden Organismen erfolgte anschliessend an die Probenahme an Ort und Stelle. Die beiden Sammelproben wurden unabhängig voneinander untersucht und protokolliert. Für jedes Protokoll wurden in der Regel drei oder mehr Lebendpräparate mikroskopiert. Úber das Vorhandensein oder Nichtvorkommen einer Art wurde somit aufgrund von mindestens sechs Lebendpräparaten entschieden. Als Bestimmungsliteratur dienten das Standardwerk von Kahl [9] sowie Arbeiten von Bick [3] und Nusch [13].

\subsection{Berechnung des Artenvorkommens}

Damit die Berechnung des Artenvorkommens sinnvoll wird, muss dafür gesorgt sein, dass das Vorkommen der verschiedenen Arten innerhalb desselben Umweltparameters miteinander vergleichbar wird. Dies ist nur möglich, wenn die Häufigkeitssumme aller 10 Klassen für jede Art den gleichen Wert, z.B. 1, beträgt. Mit der folgenden Formel wurde die relative Häufigkeit der Arten in den einzelnen Klassen der Bezugsparameter errechnet:

$$
C_{i}=\frac{\overbrace{A_{i} \cdot 100}^{x}}{B_{i}} \cdot \frac{1}{\sum_{i=1}^{10} \frac{A_{i}}{B_{i}}} \quad\left(\sum_{i=1}^{10} C_{i}=100 \%\right) .
$$

$A_{\mathrm{i}}$ : Anzahl Stellen der i-ten Klasse, an denen die betreffende Art vorkam.

$B_{i}$ : Gesamte Anzahl Stellen der i-ten Klasse über die ganze Schweiz.

$\mathrm{C}_{\mathrm{i}}$ : Normierte prozentuale Häufigkeit der Art in der i-ten Klasse des Bezugsparameters.

In der Formel ergibt der Faktor x den Anteil der Probenahmestellen, an denen die betreffende Art gefunden wurde, zur Gesamtheit der untersuchten Stellen dieser Klasse. Der Faktor y bringt die Häufigkeitssumme, also die Summe von $\mathrm{C}_{\mathrm{i}}$ auf 1 . Zum besseren Verständnis ist die Bildung der Häufigkeitsprozente am Beispiel von Vorticella convallaria in bezug auf den gelösten organischen Kohlenstoff (DOC) in Abb. 2 graphisch dargestellt.

\subsection{Fehlergrenzen der prozentualen Belegung}

Bei derartigen Untersuchungen ist nicht nur das Vorkommen der Art wesentlich, sondern auch, wie häufig sie in einer Parameterklasse gefunden wird. Diese Information kann als relative Belegung (in Prozenten) wiedergegeben werden. Sie berechnet sich bei Poissonverteilung der Daten nach der folgenden Formel: 

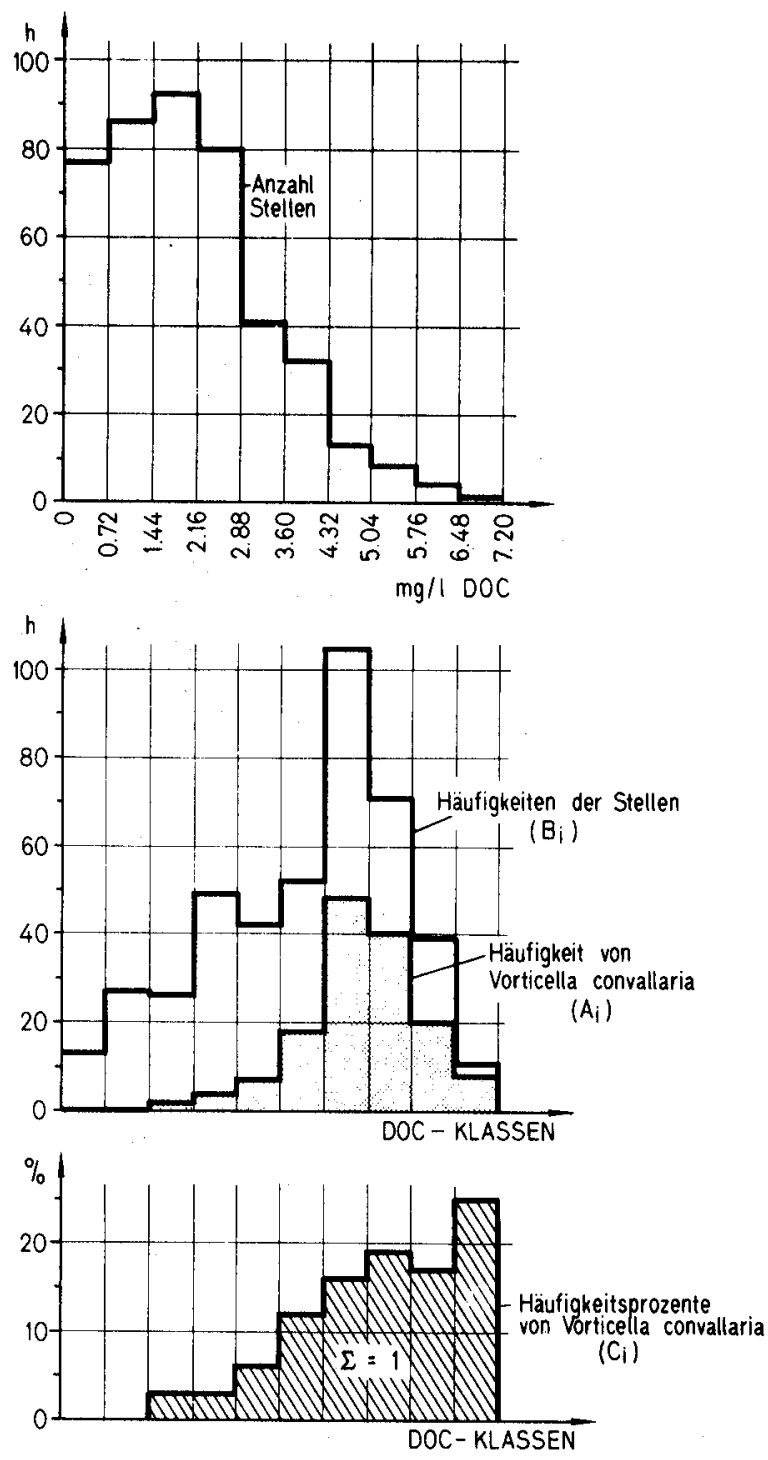

Abb. 2. Darstellung der Häufigkeitsprozente $\left(C_{i}\right)$ am Beispiel von Vorticella convallaria in bezug auf den gelösten organischen Kohlenstoff (DOC). Das oberste Histogramm zeigt als Basisinformation die Verteilung des DOC in arithmetischen Klassen. Im mittleren Teil der Darstellung ist die Häufigkeit der

Fundstellen dieser Art $\left(A_{i}\right)$ und die Häufigkeit der untersuchten Probenahmestellen $\left(B_{i}\right)$ in den 10 Klassen wiedergegeben.

Figure 2. Calculation of the frequency of occurrence of a species as a function of parameter value (using Vorticella convallaria and DOC as an example). The top diagram shows the distribution of DOC concentration on an arithmetic scale. The reason for this picture is given in chapter 2.2. Curve B (in the middle) shows the number of stations at which the DOC concentration fell within a given range or class. Curve A shows the number of stations in a given class at which $V$. convallaria was found. Curve $C$ shows the percentage of stations in a given class at which $V$. convallaria was found. 


$$
\left.Z_{i}=\frac{A_{i} \cdot 100}{B_{i}} \pm\left[\frac{A_{i}}{B_{i} \cdot \sqrt{2}} \cdot\left(\frac{1}{\sqrt{A_{i}}}+\frac{1}{\sqrt{B_{i}}}\right) \cdot 100\right]^{l}\right)
$$

$A_{\mathrm{i}}$ : Anzahl der Stellen der i-ten Klasse, an denen die untersuchte Art vorkam.

$B_{i}$ : Anzahl der Stellen der i-ten Klasse gesamthaft.

$\mathrm{Z}_{\mathrm{i}}$ : Belegung in Prozenten.

Die Resultate der Belegungsberechnung sind in Tabelle 3 zusammengestellt.

\section{Resultate}

\subsection{Artenvorkommen}

Von den rund 70 angetroffenen Ciliatenarten wurden 33 der häufig vorkommenden Arten ausgewählt. In Tabelle 1 sind diese Arten aufgelistet; die hinter den Artnamen in eckigen Klammern stehende Zahl bezieht sich auf die Abbildungsnummern der Vorkommensdarstellungen. In diesen Darstellungen wird das Vorkommen von 20 Arten mit Hilfe eines Hell-Dunkel-Rasters (Abb.3 a-t) aufgezeichnet. Verschiedene Spezies, die keine besonderen Vorkommenscharakteristiken aufwiesen, als Beispiel hierfür dient die relativ häufige Art Glaucoma scintillans (Abb.17), sind nicht abgebildet. Andere Arten wurden nur an wenigen Stellen gefunden, so dass sie sich für eine Verarbeitung in dieser Art nicht mehr eigneten. In den Vorkommensdarstellungen ist die Anzahl der Fundorte in runden Klammern hinter dem Artnamen vermerkt. Unter den Darstellungen sind eine Tabelle mit den Klassengrenzen und ein skaliertes Rastermuster, das die Abstufung der Vorkommenshäufigkeit erklärt, eingeschoben.

In der Anordnung der Bezugsparameter der Vorkommensdarstellungen sind weder ein kausaler oder funktioneller Bezug noch eine rangmässige Wertung beabsichtigt worden.

1) Erklärung der Fehlergrenzen: Aus den Gesetzen der Fehlerrechnung und bei Annahme einer Poissonverteilung kann die Streuungsberechnung folgendermassen hergeleitet werden:

Mittelwert $(\mu)=\operatorname{Varianz}\left(\mathrm{s}^{2}\right)$.

Relativer Fehler: $\frac{\mathrm{s}}{\mu}=\frac{\sqrt{\mu}}{\mu}=\frac{1}{\sqrt{\mu}} ;$ dies entspricht $\frac{1}{\sqrt{\mathrm{A}_{\mathrm{i}}}}$ oder $\frac{1}{\sqrt{\mathrm{B}_{\mathrm{i}}}}$.

Absoluter Fehler: $\frac{A_{i}}{B_{i}} \cdot$ relativer Fehler (additiv bei Division)

$$
=\frac{A_{i}}{B_{i}} \cdot\left(\frac{1}{\sqrt{A_{i}}}+\frac{1}{\sqrt{B_{i}}}\right) \text {. }
$$

Falls man annimmt, dass sich die Fehler gegenseitig ausgleichen, wird zur Berechnung der Streuung des Mittelwerts mit Faktor $\frac{1}{\sqrt{2}}$ multipliziert. 
Tabelle 1. In dieser Arbeit aufgeführte Ciliatenarten. Die hinter dem Artnamen in runden Klammern stehende Zahl bezieht sich auf die Abbildungsnummer.

Table 1. Species of ciliates discussed in this paper. The number in brackets refers to the figure containing data for that species.

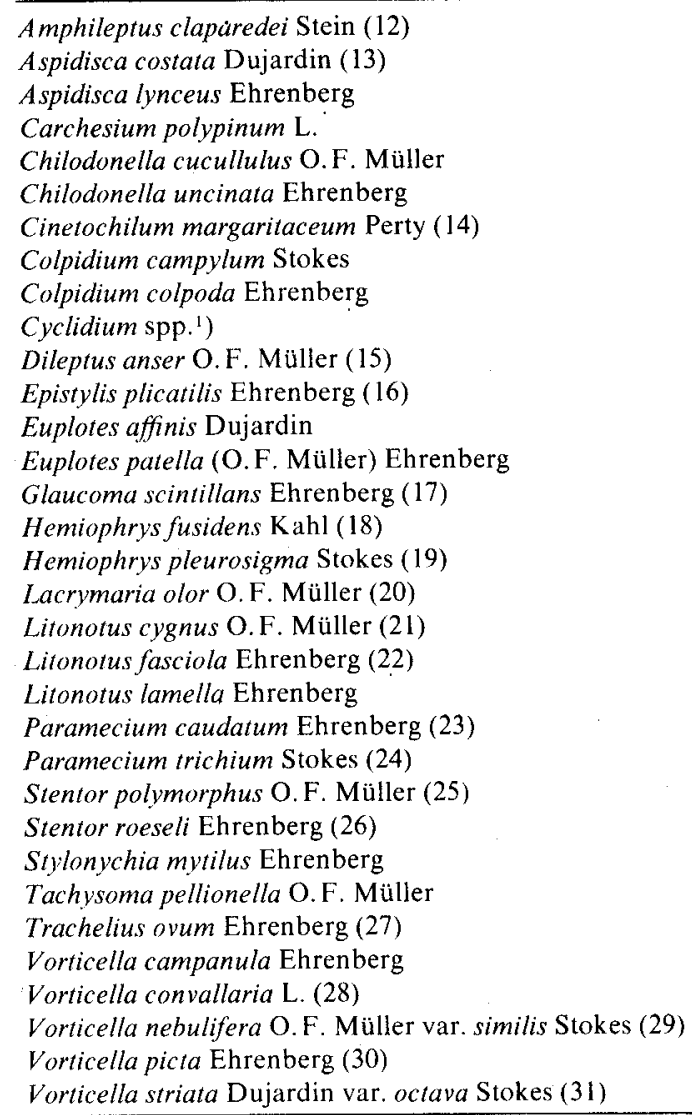

1) Unter Cyclidium spp. wurden die beiden Arten Cyclidium citrullus und Cyclidium glaucoma zusammengefasst.

\begin{tabular}{|c|c|c|c|c|c|c|c|c|c|c|c|}
\hline Klasse & 1 & 2 & 3 & 4 & 5 & 6 & 7 & 8 & 9 & 10 & \\
\hline$P_{\text {filtr. }}$ & 0.002 & 0.004 & 0.009 & 0.019 & 0.040 & 0.084 & 0.178 & 0.375 & 0.793 & 1.674 & 3.536 \\
\hline$P_{\text {roh }}$ & 0.005 & 0.010 & 0.019 & 0.038 & 0.074 & 0.144 & 0.282 & 0.553 & 1.084 & 2.123 & 4.158 \\
\hline$P_{\text {part. }}$ & 0.001 & 0.002 & 0.004 & 0.007 & 0.014 & 0.028 & 0.055 & 0.107 & 0.208 & 0.405 & 0.790 \\
\hline $\mathrm{N}_{\text {total }}$ & 0.25 & 0.36 & 0.52 & 0.76 & 1.10 & 1.59 & 2.30 & 3.32 & 4.81 & 7.00 & 10.07 \\
\hline $\mathrm{Kj}-\mathrm{N}$ & 0.02 & 0.03 & 0.06 & 0.10 & 0.17 & 0.29 & 0.49 & 0.83 & 1.14 & 2.40 & 4.09 \\
\hline $\mathrm{NO}_{3}-\mathrm{N}$ & 0.14 & 0.21 & 0.31 & 0.47 & 0.71 & 1.07 & 1.61 & 2.42 & 3.65 & 5.50 & 8.30 \\
\hline DOC & 0.3 & 0.4 & 0.5 & 0.7 & 1.0 & 1.3 & 1.9 & 2.6 & 3.7 & 5.2 & 7,2 \\
\hline $\mathrm{Ca}^{++}$ & 4 & 6 & 9 & 13 & 19 & 28 & 42 & 61 & 91 & 134 & 198 \\
\hline Leitf. & 25 & 36 & 51 & 73 & 105 & 150 & 215 & 308 & 441 & 632 & 905 \\
\hline Temp. & 2.6 & 3.3 & 4.1 & 5.1 & 6.3 & 7.9 & 9.9 & 12.4 & 15.5 & 19.4 & 24.2 \\
\hline
\end{tabular}


Amphileptus claparedei (35)

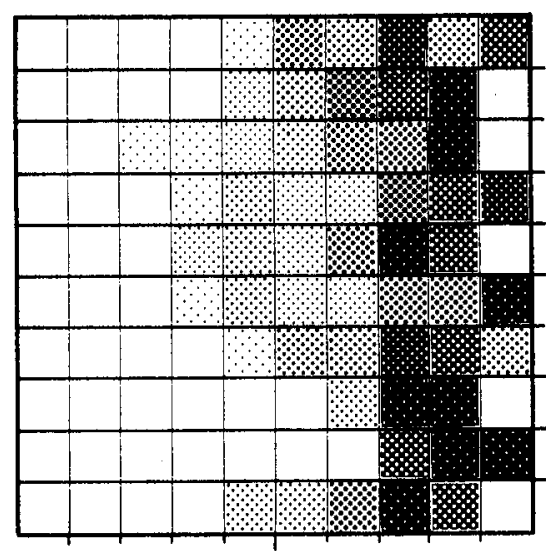

a
Aspidisca costata (33)

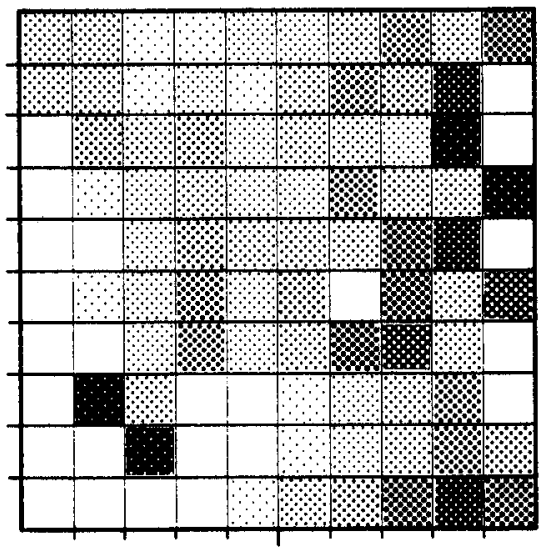

b
Cinetochilum margaritaceum (60)

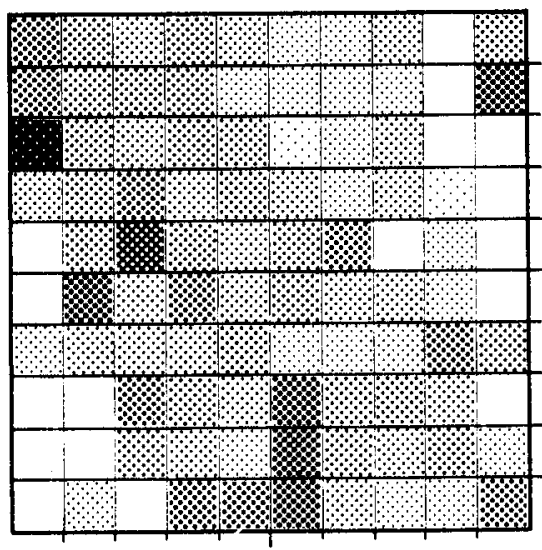

c
$P_{\text {fittr. }}$
$\mathrm{P}_{\text {roh }}$
$\mathrm{P}_{\text {part. }}$
$\mathrm{N}_{\text {total }}$
$\mathrm{Kj}-\mathrm{N}$
$\mathrm{NO}_{3}-\mathrm{N}$
$\mathrm{DOC}^{-}$
$\mathrm{Ca}^{++}$
Leitf.
Temp.

(a)
Dileptus anser (17)

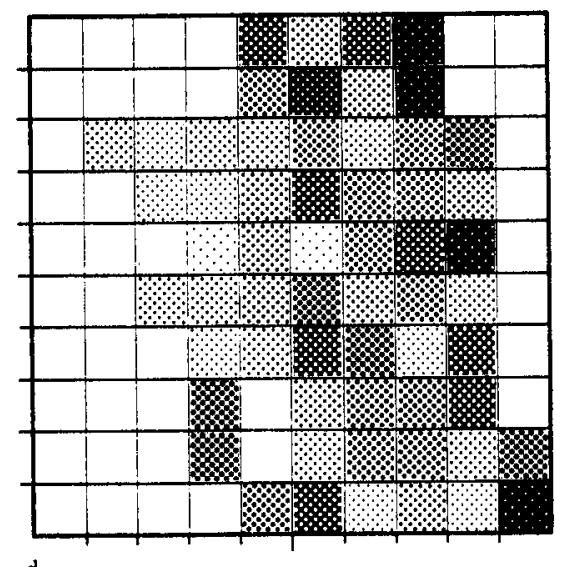

d

$P_{\text {filtr. }}$
$\mathrm{P}_{\text {roh }}$
$\mathrm{P}_{\text {part. }}$
$\mathrm{N}_{\text {total }}$
$\mathrm{Kj}-\mathrm{N}$
$\mathrm{NO}_{3}-\mathrm{N}$
$\mathrm{DOC}$
$\mathrm{Ca}$
Leitf.
Temp.

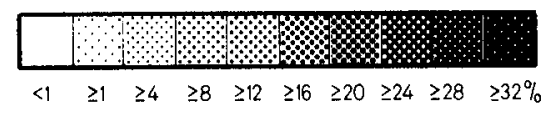

Abb. 3 a-d. Vorkommensdarstellungen von 20 Ciliatenarten im Spektrum von 10 abiotischen Faktoren. Die Vorkommenshäufigkeit in Prozenten ist durch ein Hell-Dunkel-Rastermuster wiedergegeben. Das Ablesen der ungefähren Häufigkeit wird durch ein auf jeder Doppelseite unten abgedrucktes skaliertes Muster ermöglicht. Die Klassengrenzen der 10 Parameter sind ebenfalls auf jeder Doppelseite in einer Tabelle angegeben.

Figure $3 \mathrm{a}$-d. Occurrence of 20 ciliate species as a function each of 10 abiotical factors. The frequency occurrence of each species is related to the density of dots (scale accompanies figures). The limit of parameter values included in a given class are given in tables accompanying the figures. 
Epistylis plicatilis (66)

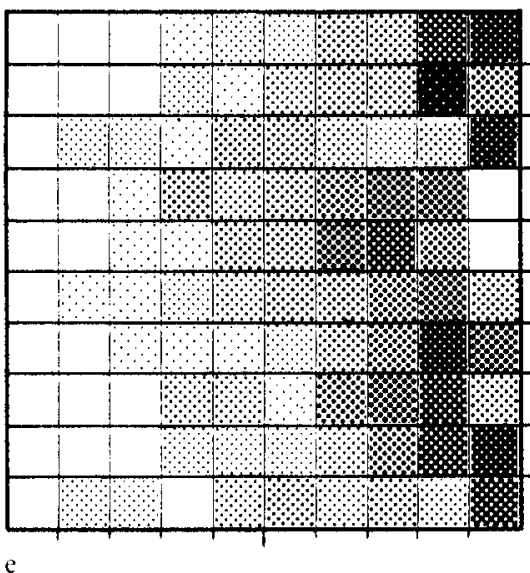

Hemiophrys fusidens (73)

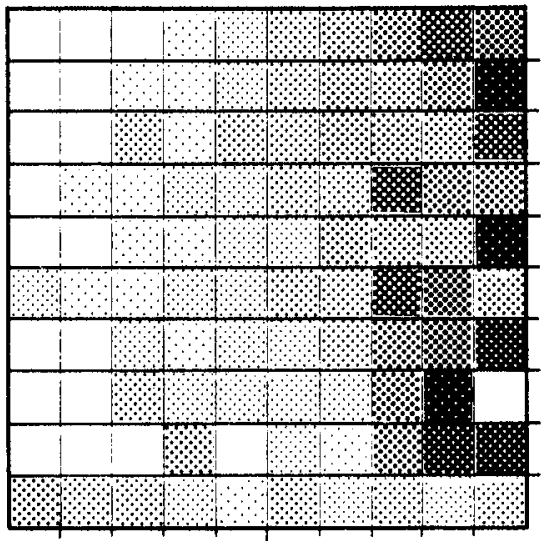

g
Glaucoma scintillans (144)

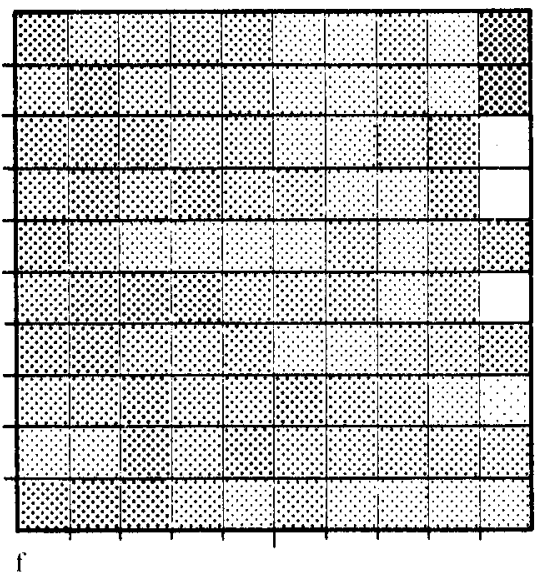

Hemiophrys pleurosigma (35)

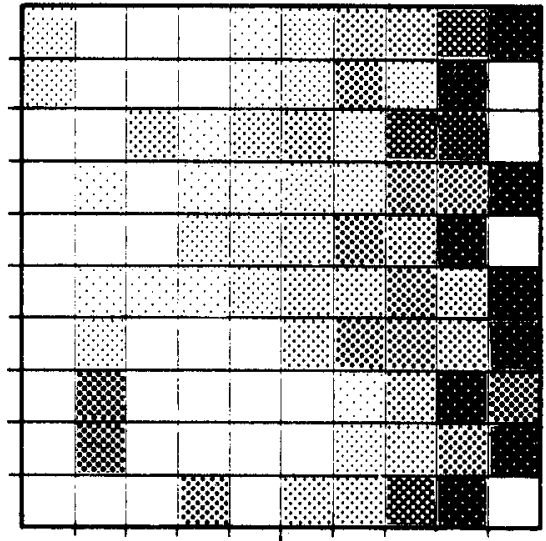

h

Abb. 3 e-h

Figure $3 \mathrm{e}-\mathrm{h}$

\begin{tabular}{|c|c|c|c|c|c|c|c|c|c|c|c|}
\hline Klasse & 1 & 2 & 3 & 4 & 5 & 6 & 7 & 8 & 9 & 10 & \\
\hline$P_{\text {filtr. }}$ & 0.002 & 0.004 & 0.009 & 0.019 & 0.040 & 0.084 & 0.178 & 0.375 & 0.793 & 1.674 & 3.536 \\
\hline$P_{\text {roh }}$ & 0.005 & 0.010 & 0.019 & 0.038 & 0.074 & 0.144 & 0.282 & 0.553 & 1.084 & 2.123 & 4.158 \\
\hline$P_{\text {part }}$ & 0.001 & 0.002 & 0.004 & 0.007 & 0.014 & 0.028 & 0.055 & 0.107 & 0.208 & 0.405 & 0.790 \\
\hline $\mathrm{N}_{\text {total }}$ & 0.25 & 0.36 & 0.52 & 0.76 & 1.10 & 1.59 & 2.30 & 3.32 & 4.81 & 7.00 & 10.07 \\
\hline $\mathrm{Kj}-\mathrm{N}$ & 0.02 & 0.03 & 0.06 & 0.10 & 0.17 & 0.29 & 0.49 & 0.83 & 1.14 & 2.40 & 4.09 \\
\hline $\mathrm{NO}_{3}-\mathrm{N}$ & 0.14 & 0.21 & 0.31 & 0.47 & 0.71 & 1.07 & 1.61 & 2.42 & 3.65 & 5.50 & 8.30 \\
\hline DOC & 0.3 & 0.4 & 0.5 & 0.7 & 1.0 & 1.3 & 1.9 & 2.6 & 3.7 & 5.2 & 7.2 \\
\hline $\mathrm{Ca}^{++}$ & 4 & 6 & 9 & 13 & 19 & 28 & 42 & 61 & 91 & 134 & 198 \\
\hline Leitf. & 25 & 36 & 51 & 73 & 105 & 150 & 215 & 308 & 441 & 632 & 905 \\
\hline Temp. & 2.6 & 3.3 & 4.1 & 5.1 & 6.3 & 7.9 & 9.9 & 12.4 & 15.5 & 19.4 & 24.2 \\
\hline
\end{tabular}


Lacrymaria olor (40)

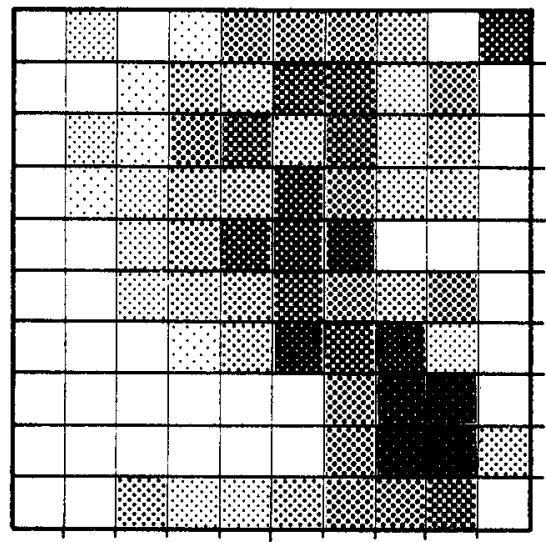

Litonotus fasciola (54)

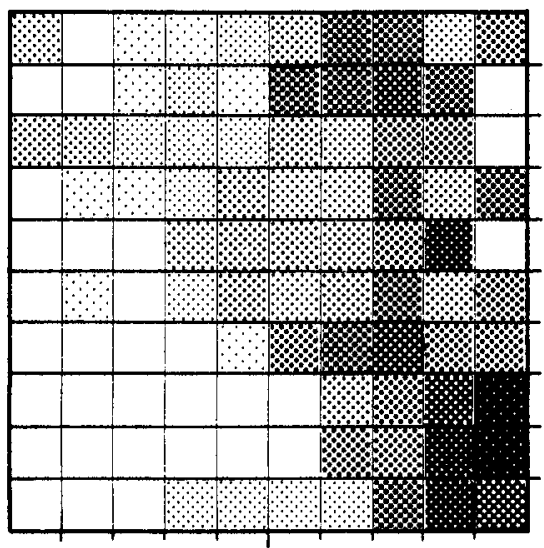

k

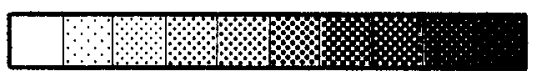

$<1 \geq 1 \geq 4 \geq 8 \geq 12 \geq 16 \geq 20 \geq 24 \geq 28 \geq 32 \%$

Abb. 3 i-1

Figure $3 \mathrm{i}-1$
Litonotus cygnus (122)

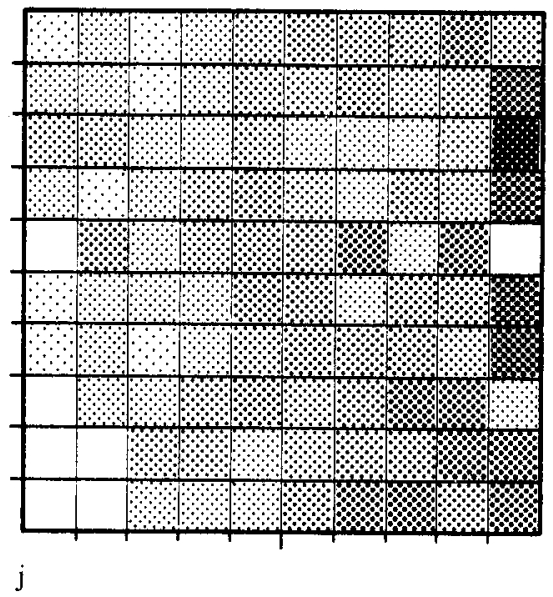

Paramecium caudatum (39)

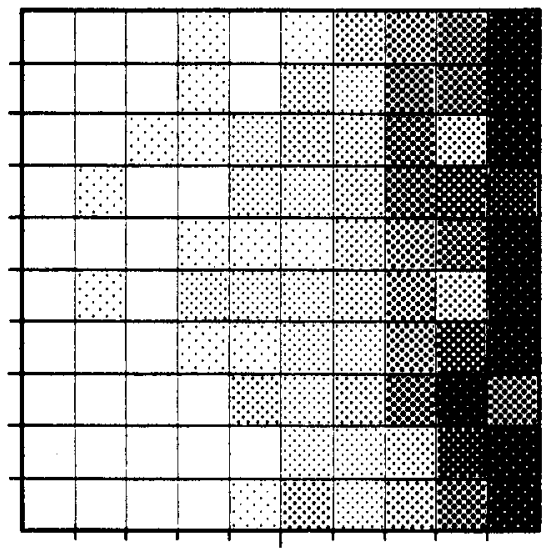

1
$P_{\text {filtr. }}$
$P_{\text {roh }}$
$P_{\text {part. }}$
$\mathrm{N}_{\text {total }}$
$\mathrm{Kj}-\mathrm{N}$
$\mathrm{NO}_{3}-\mathrm{N}$
$\mathrm{DOC}$
$\mathrm{Ca}{ }^{++}$
Lettf.
Temp. 
Paramecium trichium (39)

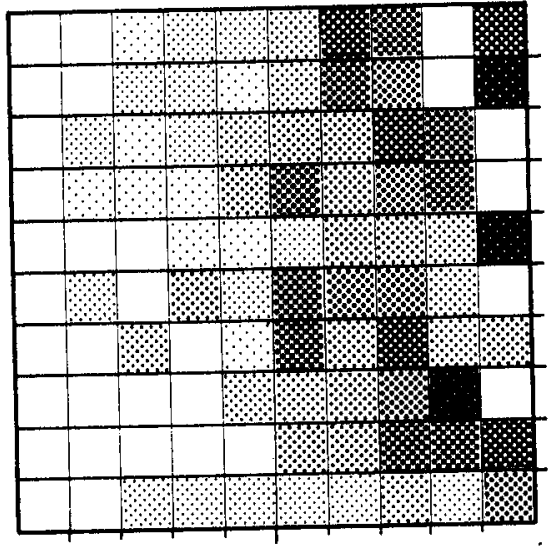

m

Stentor roeseli (56)

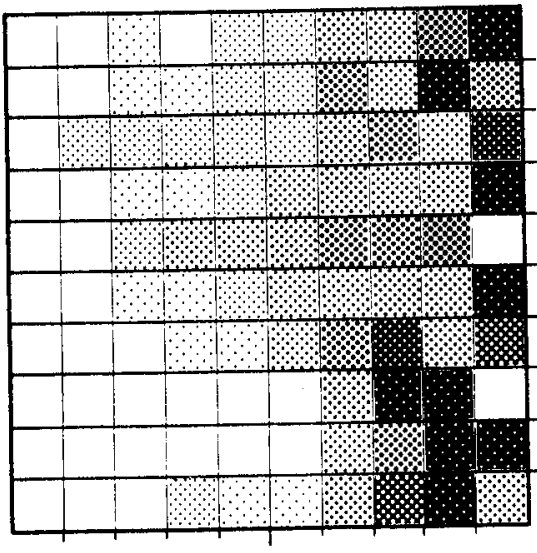

o
Stentor polymorphus (59)

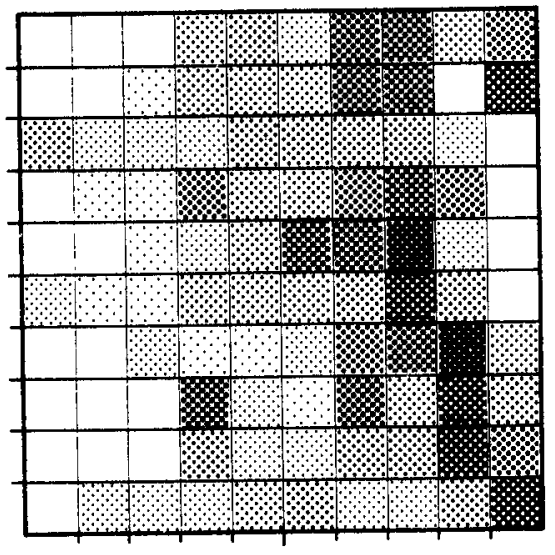

n

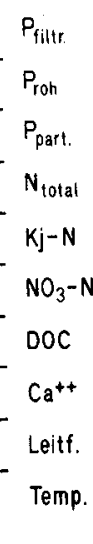

Trachelius ovum (88)

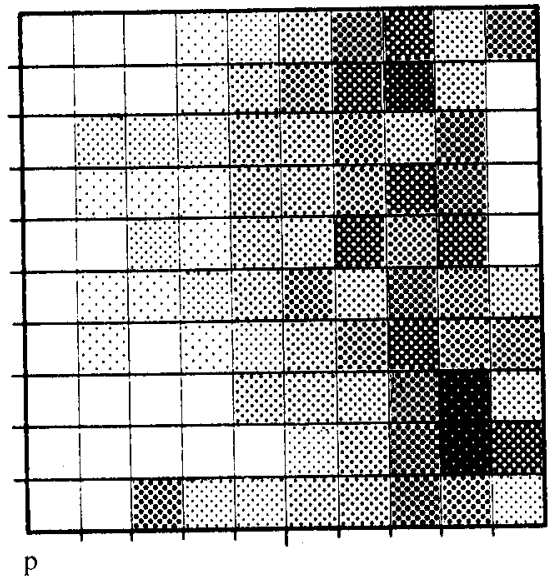

Abb. $3 \mathrm{~m}-\mathrm{p}$

Figure $3 \mathrm{~m}-\mathrm{p}$

\begin{tabular}{|c|c|c|c|c|c|c|c|c|c|c|c|}
\hline Klasse & 1 & 2 & 3 & 4 & 5 & 6 & 7 & 8 & 9 & 10 & \\
\hline$P_{\text {filtr. }}$ & 0.002 & 0.004 & 0.009 & 0.019 & 0.040 & 0.084 & 0.178 & 0.375 & 0.793 & 1.674 & 3.536 \\
\hline $\mathrm{P}_{\text {roh }}$ & 0.005 & 0.010 & 0.019 & 0.038 & 0.074 & 0.144 & 0.282 & 0.553 & 1.084 & 2.123 & 4.158 \\
\hline$P_{\text {part }}$ & 0.001 & 0.002 & 0.004 & 0.007 & 0.014 & 0.028 & 0.055 & 0.107 & 0.208 & 0.405 & 0.790 \\
\hline $\mathbf{N}_{\text {total }}$ & 0.25 & 0.36 & 0.52 & 0.76 & 1.10 & 1.59 & 2.30 & 3.32 & 4.81 & 7.00 & 10.07 \\
\hline $\mathrm{Kj}-\mathrm{N}$ & 0.02 & 0.03 & 0.06 & 0.10 & 0.17 & 0.29 & 0.49 & 0.83 & 1.14 & 2.40 & 4.09 \\
\hline $\mathrm{NO}_{3}-\mathrm{N}$ & 0.14 & 0.21 & 0.31 & 0.47 & 0.71 & 1.07 & 1.61 & 2.42 & 3.65 & 5.50 & 8.30 \\
\hline DOC & 0.3 & 0.4 & 0.5 & 0.7 & 1.0 & 1.3 & 1.9 & 2.6 & 3.7 & 5.2 & 7.2 \\
\hline $\mathrm{Ca}^{++}$ & 4 & 6 & 9 & 13 & 19 & 28 & 42 & 61 & 91 & 134 & 198 \\
\hline Leitf. & 25 & 36 & 51 & 73 & 105 & 150 & 215 & 308 & 441 & 632 & 905 \\
\hline Temp. & 2.6 & 3.3 & 4.1 & 5.1 & 6.3 & 7.9 & 9.9 & 12.4 & 15.5 & 19.4 & 24.2 \\
\hline
\end{tabular}


Vorticella convallaria (148)

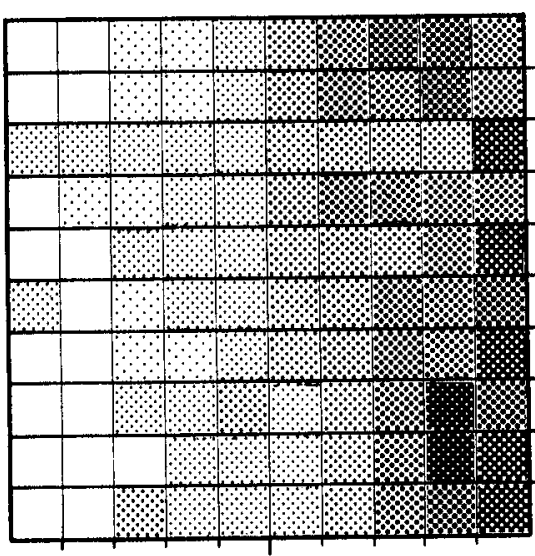

q

Vorticella picta (40)

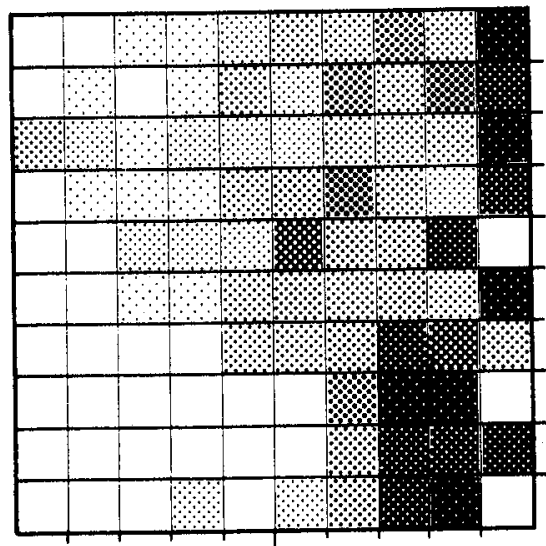

S

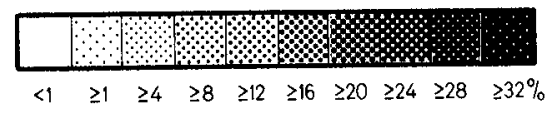

Abb. $3 \mathrm{q}-\mathrm{t}$

Figure $3 \mathrm{q}-\mathrm{t}$
Vorticella nebulifera (48)

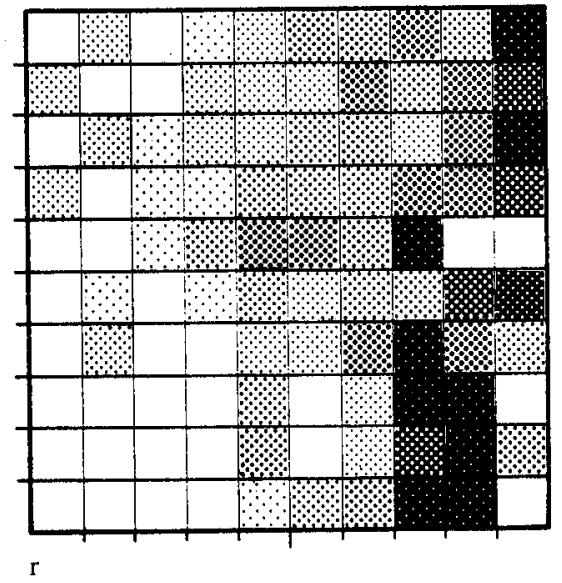

Vorticella striata (81)

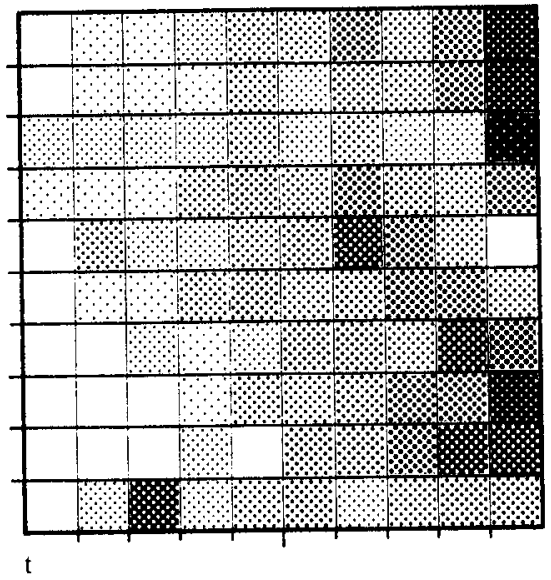




\subsection{Präferenz}

Als Versuch wird das Vorkommen aller 33 Arten im Spektrum des gelösten organischen Kohlenstoffs (DOC) noch etwas näher beleuchtet. Um besonders häufige Vorkommen abzugrenzen, ist in Tabelle 2 neben dem Fundbereich ein Präferenzbereich angegeben, dessen untere Grenze willkürlich bei einer Häufigkeit von $20 \%\left(C_{i}\right)$ pro Klasse angenommen wurde. Diese Angaben über den bevorzugten Vorkommensbereich haben jedoch nur für Fliesswasserzustände, wie sie in der Schweiz herrschen, Gültigkeit, da bei vielen Arten die obere Grenze mit der höchsten gefundenen DOC-Konzentration zusammenfällt. Es muss deshalb angenommen werden, dass mindestens bei den beiden Spezies der Gattung Hemiophrys (Abb.3g und $3 \mathrm{~h}$ ), bei Paramecium caudatum (Abb.31) und Vorticella convallaria (Abb.3q) eine Verschiebung des Präferenzbereichs und somit auch des Vorkommensbereichs in höhere Klassen bei noch grösseren DOC-Konzentrationen erfolgen würde.

\subsection{Streuung und Belegung der Arten in den Parameterklassen}

In Tabelle 3 ist die relative Belegung $Z$ und ihre Streuung (siehe Kapitel 2.5) für alle 10 Parameter aufgeführt, und zwar jeweils für diejenige Klasse, welche die «höchste» Belegung aufweist. Als Massnahme, die Signifikanz der Fehlerangaben zu verbessern, musste in der betreffenden Klasse die untersuchte Art mindestens fünfmal gefunden worden sein. Dies hat zur Folge, dass die Angaben in Tabelle 3 einen etwas anderen Schwerpunkt des Vorkommens gegenüber den Vorkommensdarstellungen (Abb.3 a-t) aufweisen können. In dieser Tabelle werden jedoch in erster Linie Streuungsangaben gemacht, die bei den Vorkommensdarstellungen nicht durchgeführt werden konnten. Zudem zeigt diese Tabelle eine weitere Möglichkeit, Indikatororganismen zu evaluieren. Dies wiederum nur in den von uns untersuchten Parametergrössenordnungen.

\section{Der Einfluss weiterer, nicht anthropogen bedingter Parameter auf das Vorkommen von Ciliaten}

Die Interpretation der Resultate hat unter Berücksichtigung von weiteren Parametern bzw. Einflüssen zu geschehen. Welche Auswirkung üben zum Beispiel die Strömungsgeschwindigkeit und die Jahreszeit auf das Vorkommen von Ciliaten aus?

\subsection{Die Strömungsgeschwindigkeit}

Eine weitere in unseren autökologischen Untersuchungen nicht berücksichtigte Grösse ist die Fliessgeschwindigkeit. Ruttner [16] beschreibt in seinem Lehrbuch das Leben der Ciliaten und anderer mikroskopisch kleiner Lebewesen in der Strömungsgrenzschicht. Das Leben der Organismen in der Grenzschicht wurde durch Ambühl [1] exakt beschrieben. Nach dieser Arbeit kann die Grenzschicht die Dicke von einigen Millimetern erreichen. Durch das Absinken der Fliessgeschwindigkeit gegen Null, bei Annäherung an den Boden, wird den Ciliaten der Aufenthalt im Fliesswasser auch ohne morphologische Anpassungen ermöglicht. Für Mikroorga- 
Tabelle 2. Artenvorkommen, bezogen auf den gelösten organischen Kohlenstoff (DOC). Für den

Präferenzbereich wurde willkürlich die Häufigkeit von $20 \%$ als untere Schranke angenommen.

Table 2. Occurrence of species as a function of dissolved organic carbon (DOC). For the optimum range a lower limit of $20 \%$ frequency was assumed.

\begin{tabular}{|c|c|c|c|c|c|}
\hline Art & $\begin{array}{l}\text { Präferenz- } \\
\text { bereich in } \\
\text { mg DOC/l }\end{array}$ & $\begin{array}{l}\text { Fund- } \\
\text { bereich in } \\
\text { mg DOC/l }\end{array}$ & Art & $\begin{array}{l}\text { Präferenz- } \\
\text { bereich in } \\
\text { mg DOC } / 1\end{array}$ & $\begin{array}{l}\text { Fund- } \\
\text { bereich in } \\
\text { mg DOC } / 1\end{array}$ \\
\hline Amphileptus claparedei & $2,6-5,1$ & $:, 7-7,2$ & Lacrymaria olor & $1,0-3,6$ & $0,7-7.2$ \\
\hline Aspidisca costata & $1,9-3,6$ & $0,5-1,4$ & Litonotus cygnus & Keiner & $0,5-5,1$ \\
\hline A spidisca lynceus & Keiner & $0,3-7,2$ & Litonotus fasciola & $1,9-3,6$ & $0,7-7,2$ \\
\hline Carchesium polypinum & Keiner $^{2}$ ) & $0,4-7,2$ & Litonotus lamella & Keiner & $0,3-7,2$ \\
\hline Chilodonella cucullulus & $5,2-7,2$ & $0,3-7,2$ & Paramecium caudatum & $3,7-7,2$ & $0,7-7,2$ \\
\hline Chilodonella uncinata & Keiner & $0,3-7,2$ & Paramecium trichium & $1,0-3,6$ & $0,5-7,2$ \\
\hline Cinetochilum margaritaceum & Keiner & $0,3-7,2$ & Stentor polymorphus & $2,6-5,1$ & $0,5-7,2$ \\
\hline Colpidium campylum & $5,2-7,2$ & $0,4-7,2$ & Stentor roeseli & $2,6-7,2$ & $0,7-7,2$ \\
\hline Colpidium colpoda & ) & $0,4-5,2$ & Stylonychia mytilus & $2,6-7,2$ & $0,5-7,2$ \\
\hline Cyclidium spp. & $3,7-5,1$ & $0,3-7,2$ & Tachysoma pellionella & Keiner & $0,3-7,2$ \\
\hline Dileptus anser & l) & $0,7-5,1$ & Trachelius ovum & $2,6-3,6$ & $0,4-7,2$ \\
\hline Epistylis plicatilis & $3,7-7,2$ & $0,5-7,2$ & Vorticella campanula & Keiner & $0,3-7,2$ \\
\hline Euplotes affinis & $3,7-5,1$ & $0,5-5,1$ & Vorticella convallaria & $5,2-7,2$ & $0,5-7,2$ \\
\hline Euplotes patella & $5,2-7,2$ & $0,4-7.2$ & Vorticella nebulifera & $2,6-3,6$ & $0,4-7,2$ \\
\hline Glaucoma scintillans & Keiner & $0,3-7,2$ & Vorticella picta & $2,6-5,1$ & $1,0-7,2$ \\
\hline Hemiophrys fusidens & $3,7-7,2$ & $0,5-7,2$ & Vorticella striata & $3,7-7,2$ & $0,5-7,2$ \\
\hline Hemiophrys pleurosigma & $5,2-7,2$ & $1,0-7,2$ & & & \\
\hline
\end{tabular}

1) Die Anzahl der Fundorte ist zu gering, um eine genügend gesicherte Präferenz angeben zu können. 2) Diese Art zeigt ab $2.6 \mathrm{mg}$ DOC/l eine schwache Zunahme des Vorkommens, jedoch noch keinen eindeutigen Präferenzbereich.

1) The number of sampling points where this species was found was too small; therefore no optimum range can be given.

2) This species shows a slight increase in occurrence above $2.6 \mathrm{mg}$ DOC/l but no clear optimum range. 
Tabelle 3. Streuung und Belegung in Prozenten der aufgeführten Arten im Spektrum der Bezugsparameter. Die Angaben gelten für die Klasse mit der höchsten Belegung. Dieselbe ist in kursiven Zahlen hinter der Streuung angegeben.

Table 3. The standard deviation and the percentage of the total number of occurrences in the class with the highest occurrence rate. The class (italic number) with the highest percentage of occurrence.

\begin{tabular}{|c|c|c|c|c|c|c|c|c|}
\hline Arten & $P_{\text {filtr. }}$ & $P_{\text {roh }}$ & & $P_{\text {parl. }}$ & & $\mathrm{N}_{\text {total }}$ & & $\mathrm{Kj}-\mathrm{N}$ \\
\hline Amphileptus claparedei & $22 \pm 6$ & $21 \pm 7$ & 8 & $42 \pm 22$ & 9 & $28 \pm 11$ & 9 & $25 \pm 128$ \\
\hline Aspidisca costata & $15 \pm 5 \quad 8$ & $15 \pm 4$ & 7 & $11 \pm 4$ & 4 & $18 \pm 6$ & 7 & $10 \pm 3 \quad 4$ \\
\hline Cinetochilum margaritaceum & $24 \pm 112$ & $22 \pm 10$ & 2 & $21 \pm 6$ & 4 & $22 \pm 6$ & 3 & $25 \pm 9$ \\
\hline Dileptus anser & $8 \pm 3 \quad 8$ & $8 \pm 3$ & 6 & $6 \pm 2$ & 6 & $8 \pm 3$ & 6 & $7 \pm 3$ \\
\hline Epistylis plicatilis & $50 \pm 279$ & $63 \pm 35$ & 9 & $21 \pm 5$ & 5,6 & $30 \pm 8$ & 8 & $30 \pm 138$ \\
\hline Glaucoma scintillans & $55 \pm 271$ & $52 \pm 18$ & 2 & $50 \pm 25$ & 9 & $47 \pm 11$ & 2,4 & $50 \pm 239$ \\
\hline Hemiophrys fusidens & $50 \pm 279$ & $31 \pm 10$ & 8 & $26 \pm 7$ & 7,8 & $47 \pm 11$ & 8 & $35 \pm 158$ \\
\hline Hemiophrys pleurosigma & $19 \pm 6 \quad 8$ & $63 \pm 35$ & 9 & $20 \pm 8$ & 8 & $24 \pm 10$ & 9 & $36 \pm 189$ \\
\hline Lacrymaria olor & $15 \pm 5 \quad 5$ & $15 \pm 4$ & 6 & $14 \pm 4$ & 5,7 & $19 \pm 5$ & 6 & $14 \pm 47$ \\
\hline Litonotus cygnus & $50 \pm 279$ & $40 \pm 8$ & 7 & $33 \pm 6$ & 5 & $41 \pm 10$ & 5 & $43 \pm 209$ \\
\hline Litonotus fasciola & $25 \pm 6 \quad 7$ & $26 \pm 9$ & 8 & $23 \pm 9$ & 8 & $32 \pm 9$ & 8 & $16 \pm 5$ \\
\hline Paramecium caudatum & $24 \pm 7$ & $23 \pm 10$ & 8 & $30 \pm 11$ & 8 & $28 \pm 11$ & 9 & $43 \pm 20$ \\
\hline Paramecium trichium & $21 \pm 5 \quad 7$ & $21 \pm 5$ & 7 & $20 \pm 8$ & 8 & $18 \pm 5$ & 6 & $25 \pm 128$ \\
\hline Stentor polymorphus & $27 \pm 7 \quad 8$ & $26 \pm 6$ & 7 & $18 \pm 5$ & 5 & $26 \pm 8$ & 8 & $30 \pm 138$ \\
\hline Stentor roeseli & $26 \pm 6 \quad 7$ & $63 \pm 35$ & 9 & $27 \pm 10$ & 8 & $27 \pm 8$ & 7 & $19 \pm 57$ \\
\hline Trachelius ovum & $46 \pm 108$ & $46 \pm 13$ & 8 & $42 \pm 22$ & 8 & $47 \pm 11$ & 8 & $50 \pm 23$ \\
\hline Vorticella convallaria & $73 \pm 158$ & $75 \pm 40$ & 9 & $55 \pm 11$ & 7 & $67 \pm 15$ & 7 & $64 \pm 27$ \\
\hline Vorticella nebulifera & $22 \pm 6 \quad 8$ & $23 \pm 6$ & 7 & $17 \pm 5$ & 7 & $25 \pm 7$ & 8 & $30 \pm 138$ \\
\hline Vorticella picta & $20 \pm 6$ & $22 \pm 5$ & 7 & $14 \pm 4$ & 7 & $22 \pm 7$ & 7 & $19 \pm 5$ \\
\hline Vorticella striata & $34 \pm 7 \quad 7$ & $32 \pm 7$ & 7 & $23 \pm 5$ & 5 & $31 \pm 9$ & 7 & $40 \pm 9$ \\
\hline
\end{tabular}

\begin{tabular}{lclllllllll}
\hline Arten & $\mathrm{NO}_{3}-\mathrm{N}$ & DOC & & $\mathrm{Ca}^{++}$ & & $\begin{array}{l}\text { Leit- } \\
\text { fähigkeit }\end{array}$ & $\begin{array}{r}\text { Tem- } \\
\text { peratur }\end{array}$ \\
\hline Amphileptus claparedei & $21 \pm 6$ & 8 & $20 \pm 5$ & 8 & $19 \pm 5$ & 9 & $16 \pm 7$ & 10 & $14 \pm 3$ & 8 \\
Aspidisca costata & $17 \pm 6$ & 8 & $13 \pm 4$ & 8 & $13 \pm 4$ & 9 & $13 \pm 3$ & 9 & $15 \pm 5$ & 9 \\
Cinetochilum margaritaceum & $26 \pm 8$ & 2 & $26 \pm 9$ & 9 & $21 \pm 8$ & 6 & $24 \pm 8$ & 6 & $29 \pm 7$ & 6 \\
Dileptus anser & $7 \pm 3$ & 6 & $7 \pm 2$ & 7 & $6 \pm 2$ & 9 & $5 \pm 2$ & 8 & $8 \pm 3$ & 6 \\
Epistylis plicatilis & $32 \pm 10$ & 9 & $41 \pm 12$ & 9 & $22 \pm 5$ & 9 & $26 \pm 10$ & 10 & $21 \pm 6$ & 6 \\
Glaucoma scintillans & $44 \pm 10$ & 2,4 & $54 \pm 25$ & 1 & $55 \pm 15$ & 3,6 & $53 \pm 23$ & 5 & $73 \pm 34$ & 3 \\
Hemiophrys fusidens & $43 \pm 11$ & 8 & $45 \pm 24$ & 10 & $33 \pm 7$ & 9 & $28 \pm 6$ & 9 & $21 \pm 4$ & 8 \\
Hemiophrys pleurosigma & $17 \pm 6$ & 8 & $14 \pm 4$ & 8 & $22 \pm 5$ & 9 & $29 \pm 11$ & 10 & $17 \pm 6$ & 9 \\
Lacrymaria olor & $15 \pm 5$ & 6 & $18 \pm 5$ & 8 & $18 \pm 5$ & 9 & $15 \pm 3$ & 8 & $13 \pm 5$ & 9 \\
Litonotus cygnus & $41 \pm 11$ & 5 & $55 \pm 27$ & 10 & $35 \pm 6$ & 8 & $39 \pm 13$ & 10 & $35 \pm 6$ & 8 \\
Litonotus fasciola & $26 \pm 8$ & 8 & $23 \pm 6$ & 8 & $23 \pm 5$ & 9 & $23 \pm 9$ & 10 & $28 \pm 8$ & 9 \\
Paramecium caudatum & $21 \pm 6$ & 8 & $26 \pm 9$ & 9 & $18 \pm 5$ & 9 & $29 \pm 11$ & 10 & $19 \pm 6$ & 9 \\
Paramecium trichium & $15 \pm 5$ & 6 & $20 \pm 5$ & 8 & $20 \pm 5$ & 9 & $11 \pm 3$ & 8,9 & $11 \pm 3$ & 8 \\
Stentor polymorphus & $32 \pm 9$ & 8 & $33 \pm 10$ & 9 & $23 \pm 5$ & 9 & $21 \pm 5$ & 9 & $21 \pm 6$ & 6 \\
Stentor roeseli & $26 \pm 8$ & 8 & $30 \pm 7$ & 8 & $28 \pm 6$ & 9 & $24 \pm 5$ & 9 & $30 \pm 8$ & 9 \\
Trachelius ovum & $43 \pm 11$ & 8 & $44 \pm 9$ & 8 & $43 \pm 8$ & 9 & $35 \pm 7$ & 9 & $30 \pm 5$ & 8 \\
Vorticella convallaria & $70 \pm 15$ & 8 & $73 \pm 34$ & 10 & $65 \pm 11$ & 9 & $56 \pm 9$ & 9 & $67 \pm 35$ & 10 \\
Vorticella nebulifera & $35 \pm 11$ & 9 & $27 \pm 7$ & 8 & $24 \pm 6$ & 9 & $23 \pm 5$ & 9 & $21 \pm 4$ & 8 \\
Vorticella picta & $15 \pm 5$ & 6,8 & $21 \pm 6$ & 8 & $18 \pm 5$ & 9 & $14 \pm 4$ & 9 & $22 \pm 7$ & 9 \\
Vorticella striata & $35 \pm 11$ & 9 & $46 \pm 13$ & 9 & $24 \pm 6$ & 9 & $26 \pm 5$ & 9 & $25 \pm 24$ & 3 \\
\hline
\end{tabular}


nismen spielt somit die Fliessgeschwindigkeit eine untergeordnete Rolle in bezug auf ihr Vorkommen, solange wenigstens das Substrat des Gerinnebettes stabil bleibt und nicht durch Hochwasser bewegt wird. Dies ist in unseren Gebirgsgewässern relativ häufig der Fall, weshalb Probenahmestellen mit erhöhtem Wasserstand (auch Stellen des Mittellandes) einige Zeit nach dem Abklingen des Hochwassers erneut untersucht wurden. Zudem kann die für Ciliaten relevante Fliessgeschwindigkeit in unmittelbarer Substratnähe nicht gemessen werden. Auf die Ausbildung von Choriozönosen hat eine starke Schwankung der Fliessgeschwindigkeit insofern einen Einfluss, als die Ablagerung von organischem Material, dessen bakterieller Abbau die Nahrungsgrundlage der meisten Ciliaten bildet, erschwert wird. Durch die von uns praktizierte Ciliatensammelmethode (Kapitel 2.3) haben wir versucht, auch beim Vorhandensein von nur wenigen Choriotopen, wenigstens die Mehrzahl der in diesem Fliesswasserabschnitt lebenden Ciliatenarten zu finden. Wie wenig die Fliessgeschwindigkeit sich auf das Vorkommen von Ciliaten auswirkt, soll das folgende Beispiel zeigen: Im Landwasser, einem sehr schnell fliessenden Gebirgsfluss (1,8-2,4 m/s zur Zeit der Probenahme), konnte mehrfach Hemiophrys fusidens, Chilodonella cucullulus, Chilodonella uncinata, Colpidium campylum, Colpidium colpoda und Tachysoma pellionella gefunden werden. Sehr häufig trat an dieser Stelle Glaucoma scintillans auf. Carchesium polypinum, Vorticella convallaria, Pleuronema coronatum und Litonotus lamella waren weitere, vorkommende Ciliaten. Diese Beispiel soll zeigen, dass trotz hoher Fliessgeschwindigkeit in diesem Fluss Ciliatenarten gefunden wurden, die auch in einem langsam fliessenden Gewässer in dieser oder einer ähnlichen Artenzusammensetzung vorkommen. Dieser Befund spricht deutlich für die untergeordnete Rolle der Fliessgeschwindigkeit in bezug auf das Artenvorkommen.

\subsection{Die Jahreszeit}

Der Einfluss der Jahreszeit auf das Vorkommen der verschiedenen Ciliatenarten ist weitgehend unbekannt. Wie Heuss [8] für einige Arten zeigt, kann die jahreszeitliche Verteilung derselben Art von Gewässer zu Gewässer und von Station zu Station variieren. Der Grossteil der Ciliaten kommt jedenfalls ganzjährig vor, aber mit unterschiedlichen Häufigkeiten im Jahresablauf.

\section{Vergleich mit autökologischen Angaben anderer Autoren}

Interessant ist der Vergleich mit den Nitratwerten aus der Arbeit Schmerenbecks [17]. In seiner Arbeit misst er sehr hohe Nitratwerte $(99,44 \mathrm{mg} \mathrm{N} / 1)$, wie sie in unseren schweizerischen Gewässern nicht vorkommen. Seine Nitratkonzentrationen beginnen mit Werten, die nicht allzusehr von unseren Höchstwerten entfernt sind. Dies gibt uns die Gelegenheit, etwas über den Einfluss des Nitrats auf das Vorkommen einiger Ciliaten auszusagen. Für das Vorkommen von Cinetochilum margaritaceum gibt er eine untere Konzentrationsgrenze von $4,5 \mathrm{mg} \mathrm{N} / \mathrm{l}$ an. Nach unserem Material liegt die untere Vorkommensgrenze bei $0,2 \mathrm{mg} \mathrm{N} / 1$. Für Epistylis plicatilis gilt als untere Konzentrationsgrenze ebenfalls $0,2 \mathrm{mg} \mathrm{N} / 1$ (Schmerenbeck: $2,7 \mathrm{mg}$ 
N/1). Bei Tachysoma pellionella und Vorticella campanula ${ }^{2}$ ) beträgt unsere tiefste Konzentration gar 0,14 mg N/1, bei Schmerenbeck $10,17 \mathrm{mg} \mathrm{N} / 1$. Unter diesen vier Ciliaten finden sich sowohl Bakterien als auch Algenfresser; man darf deshalb wohl annehmen, dass das Nitrat in den untersuchten Grössenordnungen keinen Einfluss auf das Vorkommen von Ciliaten ausübt. Diese Feststellung gilt wahrscheinlich für alle von uns untersuchten chemischen Parameter (mit Ausnahme des DOC), da sie weder giftig sind noch die Nahrungsgrundlage bilden. Somit können nur mögliche Zusammenhänge gezeigt werden.

In der Arbeit von Heuss [8] sind leider nur die beiden physikalischen Parameter Wassertemperatur und elektrische Leitfähigkeit direkt mit unseren Befunden zu vergleichen, da ungleiche chemische Parameter gewählt wurden.

Für Chilodonella cucullulus ${ }^{3}$ ) fanden wir bezüglich Leitfähigkeit eine untere Vorkommensgrenze von $35 \mu \mathrm{S} / \mathrm{cm}^{-1}$. Heuss gibt sie mit etwa $100 \mu \mathrm{S} / \mathrm{cm}^{-1}$ an, also höher als die bei uns gefundene. Dieselbe Feststellung gilt für Litonotus cygnus (Abb.3j) mit einer unteren Limite von $151 \mu \mathrm{S} / \mathrm{cm}^{-1}$ bei Heuss und $51 \mu \mathrm{S} / \mathrm{cm}^{-1}$ in schweizerischen Fliessgewässern.

Für Trachelius ovum (Abb. $3 \mathrm{p}$ ) betrug der Temperaturbereich $4-24^{\circ} \mathrm{C}$ (Heuss: $2.7-18{ }^{\circ} \mathrm{C}$ ) und derjenige für die elektrische Leitfähigkeit $150-905 \mu \mathrm{S} / \mathrm{cm}^{-1}$ (Heuss: 469-726 $\mu \mathrm{S} / \mathrm{cm}^{-1}$ ); somit ergab sich bei unseren Untersuchungen für beide Parameter eine grössere Vorkommensspanne.

Für Tachysoma pellionella ${ }^{3}$ ) stimmen die Angaben für beide Parameter ziemlich genau überein. Die hier vorliegenden Unterschiede in der Spanne der elektrischen Leitfähigkeit sind möglicherweise darauf zurückzuführen, dass auf der einen Seite Heuss keine Gewässer mit tieferen Leitfähigkeiten untersucht hat und auf der anderen Seite dieser Parameter auf das Vorkommen von Ciliaten keinen Einfluss ausübt.

Über Vorticella nebulifera kann in ganz anderer Weise ein Vergleich angestellt werden. Wir versuchten. trotz unsicheren taxonomischen Merkmalen, diese Art von V.convallaria zu trennen, doch zeigt die Vorkommensdarstellung ein ähnliches Verteilungsbild wie dasjenige der letztgenannten Art (unter Berücksichtigung der kleineren Anzahl von Fundorten). Die Zuordnung von V. nebulifera zum Formenkreis V.convallaria, wie dies Nusch [13] anregt, scheint somit gerechtfertigt.

\section{Die Eignung der Ciliaten als Bioindikatoren}

Von verschiedenen Autoren wird die kosmopolitische Verbreitung der Ciliaten bestätigt. Von dieser Seite her gesehen wären Ciliaten mit ihrer zoogeographischen Unabhängigkeit ideale Bioindikatoren. In kleineren geographischen Grenzen kam dies auch bei unseren Untersuchungen zum Ausdruck. So fanden wir in Gebirgsgewässern dieselben Arten, wie sie auch in Flachlandgewässern auftraten.

Die andere Bedingung, die an einen brauchbaren Bioindikator geknüpft ist, betrifft seine Abhängigkeit von gewässerbelastenden Faktoren, wie sie in unseren Vorkommensdarstellungen (Abb. $3 \mathrm{a}-\mathrm{t}$ ) gezeigt werden. Ideal sind stenöke Arten, die eine

2) Diese beiden Arten sind nicht in den Vorkommensdarstellungen berücksichtigt worden.

3) Diese Art ist in der Vorkommensdarstellung nicht berücksichtigt worden. 
ausgesprochene Präferenz gegenüber einem bestimmten Gewässerzustand oder Parameter aufweisen. Bevor nun aber die einzelnen Artenvorkommen in bezug auf die abiotischen Paramter diskutiert werden, sind zu den Darstellungen noch einige allgemeine Bemerkungen notwendig. Versucht man, die in dieser Arbeit veröffentlichten Daten mit den Ergebnissen in der Literatur zu vergleichen, stösst man auf verschiedene Hindernisse. Zwar sind viele Untersuchungen an Modellfliessgewässern durchgeführt worden, doch wurden nur selten dieselben Parameter analysiert. Nach unserer Ansicht können Rinnenexperimente, wie sie zum Beispiel von Schmerenbeck [17] gemacht wurden, nicht mit Freilanduntersuchungen verglichen werden. Heuss [8] zeigt sehr schön den Unterschied zwischen Objektträgerbewüchsen und von Mischproben vom natürlichen Standort, wie sie auch von uns gemacht wurden. Vergleicht man die Vielfalt der Mikrohabitate einer natürlichen Fliesswassersohle mit ihren unzähligen Totwasserräumen mit denjenigen ausgesetzter Objektträger in einer Experimentierrinne, so ist die bei Objektträgerbewüchsen grössere Streuung der Artenzahl verständlich.

Die Rasterdarstellungen (Abb. $3 \mathrm{a}-\mathrm{t}$ ) lassen bei den meisten Ciliaten keine Vorkommensschranken in unserem Untersuchungsbereich erkennen. Dies gilt sowohl nach oben wie auch etwas vermindert nach unten im Spektrum der chemischen und physikalischen Parameter. Ausnahmen zeigen sich gehäuft beim Kalzium, der Leitfähigkeit und der Wassertemperatur. Bei diesen drei Parametern ergibt sich für den grössten Teil der untersuchten Ciliaten eine Vorkommensschranke nach unten und eine Vorkommenshäufung in den oberen Klassen. Diese Schranken sind möglicherweise nicht nur auf die Autökologie dieser Arten zurückzuführen, sondern auch auf die Art der gewählten Verteilung, da diese drei Parameter starke Häufungen in den oberen Klassen aufweisen (Abb.8-10). Bezüglich der anderen sieben Parameter zeigen die untersuchten Ciliaten im allgemeinen ein ubiquitäres Vorkommen. Dennoch weisen einige Arten in einzelnen oder mehreren oberen Klassen eindeutige Häufungen des Vorkommens auf. Dies gilt vor allem für die nachgenannten Arten: Amphileptus claparedei (Abb.3a), Epistylis plicatilis (Abb.3e), Hemiophrys fusidens (Abb.3g), H.pleurosigma (Abb.3h), Paramecium caudatum (Abb.31), P.trichium (Abb.3m), Stentor roeseli (Abb.3o) und Vorticella picta (Abb. $3 \mathrm{~s})$. Nachfolgend werden die Vorkommen der einzelnen Arten in den gefundenen Konzentrationen des gelösten organischen Kohlenstoffs (DOC) diskutiert.

Amphileptus claparedei (Abb. 3 a) zeigt in allen oberen Klassen der Nährstoff- und Belastungsparameter ein bevorzugtes Vorkommen. Im revidierten Saprobiensystem Liebmanns [11] und in den Listen Sládečeks [20] wird $A$. claparedei als $\alpha$-mesosaprobe Form geführt. Nach unseren Ergebnissen ist diese Art ebenfalls als Indikator für stärkere Belastungen einzuordnen.

Die bakterienabweidende Aspidisca costata (Abb. 3b) ist als Indikator für belastete Gewässerzustände bereits deutlich weniger geeignet. In höheren DOC-Klassen weist sie eine leichte Häufung des Vorkommens auf und kann deshalb kaum ein $\beta$ mesosaprober Anzeiger sein, wie von Liebmann vorgeschlagen wird. Da A.costata im höchsten von uns gemessenen Bereich des gelösten organischen Kohlenstoffs nicht mehr vorkam, dürfte auch die Angabe Bucks [6], der sie als $a$-bis polysaprob angibt, wenigstens für die schweizerischen Fliessgewässer nicht zutreffen. Einerseits kam diese Art bei den meisten DOC-Konzentrationen vor und zeigte auch keine 
eindeutige Präferenz, andrerseits fanden wir sie nie in höheren Abundanzen. Dies sind Hinweise, nach denen diese Form als Bioindikator ungeeignet ist.

Carchesium polypinum ${ }^{4}$ ) kommt bei uns sehr häufig vor, sowohl in den Berggewässern als auch im Fliesswasser des Flachlandes. Ihre stärkste Verbreitung findet sie bei höheren organischen Belastungen und bildet dann auch grössere weissliche Polster an Steinen des Grundes. In diesem Sinn ist C.polypinum sehr gut als Belastungsanzeiger verwendbar.

Die nächste Art, Cinetochilum margaritaceum (Abb.3c), weist keine Vorkommensschwerpunkte auf und ist deshalb als Bioindikator unbrauchbar. Auch von Sládeček [20] wird C. margaritaceum als pantosaprob bezeichnet.

Die omnivore Art Dileptus anser (Abb.3d) wurde nur an wenigen Probenahmestellen gefunden. Dadurch wird die Angabe einer Indikation unzuverlässig. Von Liebmann wird sie als oligosaprob, von Sládeček als oligo- $\beta$-mesosaprob in den Listen geführt. Diese Einstufung ist jedoch nach unseren Ermittlungen nicht haltbar, zeigt doch diese Art eher ein erhöhtes Vorkommen im mittleren Belastungsbereich.

Abb. 16 zeigt das Vorkommensspektrum von Epistylis plicatilis. Im Bereich des gelösten organischen Kohlenstoffs liegt der Vorkommensschwerpunkt dieser Art eindeutig in den stark belasteten Gewässern. Etwas weniger häufig wurde E.plicatilis aber auch in weniger bis unbelasteten Gewässern gefunden. In vergleichbarer Weise wird dieses Peritrich auch von Sládeček sowie von Bick [3], nach der 10Punkte-Methode von Zelinka und Marvan [25], eingestuft. Allerdings wird die Indikation der schwachen Belastung durch diese Autoren weniger betont.

Einige Probleme bietet Glaucoma scintillans (Abb.3f) bei der Interpretation ihrer Indikationstauglichkeit. Wird sie doch von Liebmann, Sládeček und Buck als polysaprober Anzeiger gewertet. Ein Blick auf unsere Darstellung (Abb.3f) lässt hingegen keine bevorzugten Vorkommenshäufigkeit erkennen, da sie in allen Belastungsklassen nahezu gleich stark vertreten ist. Dieser Widerspruch erklärt sich möglicherweise zum Teil mit der Eigenschaft montaner Fliessgewässer (siehe S. 136), deren Ergebnisse überwiegend in den unteren Klassen der Vorkommensdarstellungen wiedergegeben werden. Es muss deshalb angenommen werden, dass G. scintillans als bakterivorer Ciliat erst in höheren Abundanzstufen, wenn überhaupt, als Indikatororganismus tauglich ist.

Wesentlich einfacher ist Hemiophrys fusidens (Abb.3g) zu beurteilen. Buck stuft diesen Ciliaten als $a$-mesosaprob bei Präsenz ein, was mit unseren Befunden nicht vereinbar ist. Mit den Angaben Sládečeks allerdings ist unsere Vorkommensdarstellung recht gut vergleichbar. Als Prädator richtet sich das Vorkommen dieser Art nach der Frequenz seiner Beuteorganismen aus. Es muss daraus geschlossen werden, dass der Sekundärkonsument $H$.fusidens erst bei mehrfachen Funden für belastete Zustände Indikationswert besitzt.

Für Hemiophrys pleurosigma (Abb. $3 \mathrm{~h}$ ) gelten grundsätzlich dieselben Bemerkungen wie für $H$.fusidens.

Lacrymaria olor (Abb.3i) ist bei Buck ein Indikator bei Präsenz für unbelastete bis leicht belastete Gewässerzustände. Bick weitet diese Indikation bis in den $a$ -

4) Diese Art ist in den Vorkommensdarstellungen nicht berücksichtigt. 
mesosaproben Bereich aus und kommt so unseren Untersuchungsergebnissen am nächsten. L.olor wurde den schweizerischen Richtlinien [23] gemäss vorwiegend an der Schwelle zwischen anzustrebenden und zu verbessernden Zuständen von Gewässern gefunden und kann in diesem Bereich als Bioindikator eingesetzt werden.

Litonotus cygnus (Abb.3j) weist ein ähnlich undifferenziertes Verteilungsbild auf wie Glaucoma scintillans, allerdings mit einer leichten Zunahme der Vorkommenshäufigkeit bei stärkeren Belastungen. Die statistischen Berechnungen Bucks und die Angaben Sládečeks begrenzen das Vorkommen dieser Art ziemlich eng im $\beta$ mesosaproben Bereich, was wir mit Sicherheit nicht bestätigen können. Da L.cygnus im allgemeinen nur in geringen Abundanzen auftritt, ist eine Verbesserung seiner Indikationseigenschaften nicht $\mathrm{zu}$ erwarten und daher seine Indikationstauglichkeit zweifelhaft.

Die verwandte Art Litonotus fasciola (Abb. $3 \mathrm{k}$ ) hingegen zeigt mit grösserer Wahrscheinlichkeit Belastungen an. Diese Belastungen dürften im Mittel höher sein als die $2 \mathrm{mg} \mathrm{DOC/1}$, welche vom Gesetz [23] als obere Grenze vorgeschrieben werden. Die Indikationseigenschaft von Paramecium caudatum (Abb.31) wird von keinem Sachverständigen in Zweifel gezogen. Die Bestätigung von starken bis stärksten Belastungen ist jedoch erst bei häufigeren Funden gegeben.

Von Sládeček wird Paramecium trichium (Abb. $3 \mathrm{~m}$ ) als polysaprob und in schwach belasteten Gewässern nicht vorkommend dargestellt. Buck gibt ihm dieselbe Einstufung, jedoch nur bei hoher Abundanz. Unsere Untersuchungen ergaben auch in diesem Falle ein Vordringen dieser Art in leicht belastete Gewässer. Nach unseren Erfahrungen kann P.trichium als Indikator für erhöhte organische Belastungen dienen, jedoch nur bei mehrfachen Funden.

Stentor polymorphus (Abb.3n) kommt nach unseren Ermittlungen bei sämtlichen Belastungszuständen vor, weist aber bei stärkeren organischen Verschmutzungen eine grössere Häufigkeit auf. Diese Feststellung entspricht nicht derjenigen von Sládeček, der den Schwerpunkt des Vorkommens im leicht belasteten Gewässer sieht. S. polymorphus kann bei Mehrfachfunden als Indikator für erhöhte organische Frachten beigezogen werden.

Dieselbe Indikation, jedoch etwas verstärkt, gilt auch für Stentor roeseli (Abb.3o). Auch die Statistik von Buck zeigt für diese Art einen Schwerpunkt des Vorkommens am Übergang von leichten zu schwereren Belastungen.

Trachelius ovum (Abb.3p) war als Glockentierräuber am häufigsten bei grösseren Vorkommen von Carchesium polypinum oder Vorticella campanula anzutreffen. Dies wird durch eine grössere Frequenz in den höheren Belastungsklassen der Darstellungen bestätigt. Diese Art ist ebenfalls bei mehrfachen Funden für stärkere Belastungen charakteristisch. Buck und Sládeček stufen T. ovum am Übergang von schwachen zu stärkeren Belastungen ein. Diese Indikation jedoch bei Präsenz zuzulassen, wie Buck vorschlägt, ist nach unseren Ergebnissen fragwürdig, zumal Trachelius ökologisch an seine Beute gekoppelt und deshalb kein primärer Indikator ist.

Vorticella campanula ${ }^{5}$ ) ist die in schweizerischen Gewässern am häufigsten gefundene Art, die in jedem Fliessgewässertyp vorkommt. Nach Liebmann ist diese Art ein

5) Diese Art ist in der Vorkommensdarstellung nicht berücksichtigt. 
$\beta$-mesosaprober Indikator, bei Sládečková und Sládeček [21] liegt ihr Schwergewicht des Vorkommens ebenfalls im $\beta$-mesosaproben Bereich. Beide Indikationen stimmen mit unseren Erfahrungen nicht überein. Eine Massenentfaltung dieser Art konnte nur in stärker belasteten Gewässern beobachtet werden (grössere Fleckenbildungen). Eine Bedeutung als Indikator kommt dieser Art erst zu, wenn sie (als weissliche Aggregate) von Auge sichtbar ist.

Vorticella convallaria (Abb. 3q) zeigt eine sehr deutliche Vorkommensverminderung im Gefälle der Belastungsklassen. Bei der Beurteilung als Indikator muss beachtet werden, dass wir die physiologische Modifikation dieser Art (V.convallaria L. forma citrina O.F. Müller) nicht speziell unterschieden haben. Ein Indikationswert für stärkere bis starke Belastungen ist dieser Art erst bei höherer Abundanz beizumessen. Über Vorticella nebulifera (Abb.3r) kann keine Indikation abgegeben werden. Die Gründe hiefür wurden in Kapitel 5 genannt.

Bei Vorticella picta (Abb.3s) ist die Indikationseigenschaft ohne Schwierigkeit aus der Vorkommensdarstellung herauszulesen, da sie eindeutig in stärker belasteten Gewässern eine grössere Häufigkeit aufweist. Mit einiger Sicherheit darf diese Art bei nicht nur einmaligem Auftreten als Belastungsanzeiger benützt werden. Diese Indikation hat mit den Angaben Sládečeks wenig gemeinsam, der sie als oligosaprobe Form einreiht. Mit der Forma longa, wie Nusch [13] sie beschreibt und deren ökologische Daten den unseren eher entsprechen, hat die von uns gefundene Form aber vom Habitus her gesehen keine grosse Ähnlichkeit.

Vorticella striata (Abb.3t) Diese Art ist, wie Sládeček angibt, eine pantosaprobe Form, die aber in stärker organisch belasteten Gewässern eine Häufung des Vorkommens aufweist. Bei unseren Untersuchungen jedoch konnte sie nie in grösseren Abundanzen festgestellt werden wie z. B. V.campanula oder V.convallaria. Sie kommt deshalb bei unseren Fliesswasserzuständen als Bioindikator nicht in Frage.

In diesem Kapitel wurde versucht, den Indikationswert einiger Ciliaten in bezug auf die DOC-Konzentration herauszuarbeiten. Die relativ vielen Funde der für eine Indikation am ehesten geeigneten, das heisst genügend stenöken Arten, an Probestellen mit geringer DOC-Konzentration, zeigen erneut die Schwierigkeiten auf, die sich ergeben. wenn man mit vermindert euryöken Typen Belastungen biologisch beschreiben will. Ein nicht zu unterschätzender Faktor, der hier hineinspielt, dürfte vor allem die in den Gebirgsgewässern durchschnittlich niedere Temperatur sein. An solchen Stellen wird organisches Material, trotz der guten Sauerstoffversorgung, nur verzögert abgebaut. Meist sind solche Flussabschnitte zudem noch durch höhere Fliessgeschwindigkeit gekennzeichnet. Dadurch ist die Sedimentation der organischen Partikeln erschwert, und ein Grossteil davon wird erst im fortgeschrittenen Flusslauf abgebaut und für Ciliaten verfügbar gemacht. Dementsprechend ist auch die für ein verstärktes Ciliatenwachstum notwendige Bakterienmenge noch nicht vorhanden. Steinmann und Surbeck [22] waren überzeugt, dass für die biologische Fliesswasserbeurteilung die qualitative Untersuchung im allgemeinen genüge und die Quantität nur für einige Formen geschätzt werden müsse. An dieser Feststellung hat sich bisher nicht viel geändert, doch muss bei ciliatologischen Untersuchungen dafür die Fliessgeschwindigkeit mitberücksichtigt werden. Im ersten Moment scheint dies ein 
Widerspruch zur Aussage in Kapitel 4.1 zu sein. Tatsächlich beeinflusst die Fliessgeschwindigkeit das Vorkommen von Ciliaten, aber - und damit löst sich auch der Widerspruch - nicht direkt, sondern auf dem Umweg über weitere Faktoren, insbesondere die Korngrösse des Gewässergrundes.

Aus der Literatur ist die Abhängigkeit der Körnung des Flussbettsubstrats von der Wassermenge pro Meter Flussbreite und dem Gefälle bekannt [7] und somit auch von der Fliessgeschwindigkeit. Die Korrelierung der "Ciliatenflecken" mit dem DOC, der Fliessgeschwindigkeit und der Körnung des Fliesswassergrundes unseres Datenmaterials ergab folgende Resultate: Der DOC korrelierte gut (Korrelationskoeffizient $r=0,46, n=199$, zu 100\% gesichert verschieden von Null), die Körnung etwas schwächer ( $r=0,23, n=199$, zu 99,9\% gesichert) und die Fliessgeschwindigkeit nicht mit der Grösse der Aggregation von sessilen Ciliaten. Das Nichtkorrelieren der Fliessgeschwindigkeit mit den sessilen Ciliaten muss erwartet werden, da für diese Berechnung eine für die Ökologie der Ciliaten nichtrelevante Fliessgeschwindigkeit verwendet wurde (siehe Kapitel 4.1). Das heisst, die Ciliatenabundanz wird letztlich von der Fliessgeschwindigkeit beeinflusst, da sie bei kleineren Körnungen, die kleineren Fliessgeschwindigkeiten entsprechen, grösser ist als bei grösseren Körnungen. Dabei spielen die Spitzengeschwindigkeiten während Hochwässern eine besondere Rolle.

Am Schluss dieser Arbeit können einige richtungweisende Aspekte über die Verwendbarkeit von Ciliaten zur biologischen Gewässerbeurteilung gegeben werden. Dabei kommt man um die Feststellung nicht herum, dass nach unseren Beobachtungen Ciliaten (als Gesamtheiten) bei weitem nicht so gute Belastungsindikatoren sind, als es die bekannten früheren Literaturarbeiten postulieren [10, 11]. Einige positive Aspekte sind immerhin vorhanden und können auch ökologisch begründet werden: So erscheinen die peritrichen sessilen Arten (in erster Linie Carchesium polypinum, Vorticella campanula und - etwas vermindert - V.convallaria) als brauchbare Bioindikatoren. Bezüglich der Konzentration organischer Belastungsparameter sind sie recht euryök (Tab.2), korrelieren mit der Belastung aber trotzdem, wenn ihr Auftreten deutlich ist. Das Ausmass ihrer Aggregatbildung hängt von der Intensität des bakteriellen Abbaus von organischem Material ab, von der seit dem letzten Hochwasser verstrichenen Zeit sowie von der mittleren Fliessgeschwindigkeit. Ferner dürfte die Abbaugeschwindigkeit eines Sessilen-Aggregats direkt und indirekt (durch das Bakterien- bzw. Futterangebot) von der Temperatur mitbestimmt werden.

Von den vagilen Ciliaten, die sehr verschiedene ökologische Typen umfassen, ist keine einzige Art stenök; bestenfalls sind einige als vermindert euryök zu bezeichnen (immer auf das chemische Spektrum der schweizerischen Gewässer bezogen). Trotzdem sollte mit einigen Arten eine biologische Indikation möglich sein, dann wenigstens, wenn mehrfach Funde nachgewiesen sind; die Präsenz allein genügt nicht (siehe Abb. 3a, g-i, k-o). Zum selben Schluss gelangt auch Bick [4] in seinem Vortrag über Ciliaten als Indikatororganismen bei Gewässeruntersuchungen.

Im übrigen dürfte die Zusammensetzung der an einer Probestelle gefundenen biologischen Typen (Bakterienstrudler, Bakterienweider, Algenfresser usw.) eine weitere Möglichkeit der Beurteilung erlauben. Eine eingehende diesbezügliche Analyse unseres Datenmaterials ist beabsichtigt. 


\section{Zusammenfassung}

In den Sommermonaten 1974 und 1975 wurden an 439 Stellen, verteilt auf 194 Fliessgewässer der Schweiz, Aufwuchsuntersuchungen an Ciliaten durchgeführt. Zur chemischen Analyse des Wassers wurden in der Regel zwei zeitverschobene Stichproben entnommen. Die elektrische Leitfähigkeit und die Wassertemperatur wurden am Ort der Probenahme gemessen.

Für 33 Ciliatenarten werden der Vorkommens- und Präferenzbereich, bezogen auf den gelösten organischen Kohlenstoff (DOC), angegeben. Autökologische Daten von 20 Arten werden anhand von acht chemischen und zwei physikalischen Parametern präsentiert. Aus diesen Daten ist die nur weite Anpassung vieler Ciliaten an das vorhandene Faktorenspektrum ersichtlich (Euryökie).

Für das Ciliatenvorkommen war der DOC einer der einflussreichsten untersuchten Parameter. Die Häufigkeitsverteilung einiger Ciliaten im DOC-Spektrum wird dazu benützt, grobe Indikationsvorschläge für den Belastungszustand schweizerischer Fliessgewässer zu geben. Dabei ergaben sich zum Teil Widersprüche zu den saprobiellen Angaben von Buck, Liebmann und Sládeček. Abweichende Indikationen ergaben sich für die Arten Amphileptus claparedei, Aspidisca costata, Dileptus anser, Glaucoma scintillans, Hemiophrys fusidens, Lacrymaria olor, Litonotus cygnus, Stentor polymorphus, Trachelius ovum, Vorticella campanula und V.picta.

Es wird festgestellt, das die Fliessgeschwindigkeit keinen Einfluss auf das Vorkommen von Ciliaten ausübt, wohl aber auf deren Häufigkeit. Daraus ergibt sich folgendes Postulat:

Auch in schweizerischen Fliessgewässern können Ciliaten als Bioindikatoren verwendet werden, doch muss für eine Indikation die grobe Abundanz der Art in Relation zur mittleren Fliessgeschwindigkeit gesetzt werden.

\section{SUMMARY}

The occurrence of ciliates in 'Aufwuchs' was studied at 439 sampling stations in 194 Swiss streams during the summers of 1974 and 1975. At least two water samples from each station were taken for chemical analysis; parameters measured were: total dissolved phosphate, total phosphate, particulate phosphate, total nitrogen, Kjeldahl nitrogen in unfiltered water, nitrate, dissolved organic carbon, calcium; electrical conductivity and water temperature were measured at the site.

The occurrence and optimal ranges of 20 ciliate species is described as a function each of the eight chemical and the two physical parameters (fig. $3 \mathrm{a}-\mathrm{t})$ (33 species for dissolved organic carbon, table 2$)$. Most ciliates exhibit great tolerance to variations in one or more environmental factors (are eurytopic as opposed to stenotopic).

The dissolved organic carbon (DOC) was one of the most influential parameters for ciliate occurrence; the occurrence and abundance of some ciliates in the DOC spectrum can be used as a rough indicator of pollution conditions. The occurrence of some species (Amphileptus claparedei, Aspidisca costata, Dileptus anser, Glaucoma scintillans, Hemiophrys fusidens, Lacrymaria olor, Litonotus cygnus, Stentor polymorphus, Trachelius ovum. Vorticella companula and V.picta) was found to be contrary to that expected from the saprobiological data of Buck, Liebmann and Sládeček.

The velocity of flow had no effect on the occurrence of ciliates, but did influence their abundance. Thus the following is postulated: Ciliates can be used as bioindicators in Swiss streams, but in order to establish such an index the influence of flow velocity on the species abundance must be taken into consideration. 


\section{RÉSUMÉ}

Des échantillons de périphyton recueillis durant les mois d'été 1974/75 en 439 endroits repartis le long de 194 cours d'eau suisses sont étudiés quant à leur contenu de ciliés. Les analyses chimiques, dont les paramètres enquêtés sont: phosphore total dans l'eau filtrée et non-filtrée, phosphore particulaire, azote total, azote Kjeldahl dans l'eau non-filtrée, azote nitrique, carbone organique dissous (COD) et calcium, se font généralement à partir de deux échantillons d'eau par endroit de prélèvement. La conductivité électrique et la température de l'eau sont mesurées sur place.

La présence et le milieu optimum de 33 espèces de ciliés sont décrits par rapport à la concentration respective de carbone organique dissous trouvée dans l'eau (tabl.2), et pour 20 de ces 33 espèces, la distribution de fréquence est présentée en fonction de huit paramètres chimiques et deux paramètres physiques (fig. $3 \mathrm{a}-\mathrm{t}$ ). La plupart des ciliés font preuve d'une assez grande faculté d'adaptation à des variations de un ou de plusieurs facteurs de leur environnement.

Le carbon organique dissous s'avérant être un des paramètres déterminants pour l'apparticion de ciliés, la présence ainsi que l'abondance de certaines espèces peuvent grosso modo servir d'indicateurs du degré de pollution d'une eau. Notre observation de certaines espèces (telles Amphileptus claparedei, Aspidisca costata, Dileptus anser, Glaucoma scintillans, Hemiophrys fusidens, Lacrymaria olor, Litonotus cygnus, Stentor polymorphus, Trachelius ovum, Vorticella campanula et V. picta) va à l'encontre des suppositions de Buck, Liebmann et Sládeček.

La rapidité du courant a une influence non pas sur la présence mais sur l'abondance des ciliés. Ainsi l'hypothèse suivante est avancée:

Les ciliés peuvent servir d'indicateurs de la condition biologique d'une eau courante en Suisse, il importe cependant de considérer leur nombre en relation avec la vitesse moyenne du courant à l'endroit du prélèvement afin d'obtenir un indice assez précis.

\section{VERDANKUNGEN}

Die mikroskopische Untersuchung wäre ohne Unterstützung verschiedener Leute nicht möglich gewesen. Herrn Prof. Dr. H. Ambühl, Leiter der Abteilung Limnologie, danke ich für sein Entgegenkommen, das die Ausführung der Arbeit erst ermöglichte. Die mikroskopischen Analysen und die begleitenden Untersuchungen an den Probenahmestellen wurden durch die folgenden Mitarbeiter des MaposProgramms vorgenommen: Jeannette Bigler, Gaby Fricker, Hj. Fricker, B. Horlacher, M. Rüttimann, W. Schneider und C. Sturzenegger. Die Herren E. Szabó, R. Illi und B. Ribi bearbeiteten im Laboratorium der Abteilung Limnologie die zahlreichen Wasserproben. Bei den umfangreichen statistischen Auswertungen half mir Dr. H. Bührer. Heidi Bolliger zeichnete die Häufigkeitsdarstellungen der chemischen Werte. Allen diesen Damen und Herren danke ich für ihre Mitarbeit. Dank gebührt auch den Herren Prof. Dr. H. Ambühl, Dr. P. Perret und H. Güttinger für die kritische Durchsicht und die wertvollen Vorschläge bei der Bearbeitung dieses Manuskripts.

\section{LITERATURVERZEICHNIS}

1 Ambühl, H.: Die Bedeutung der Strömung als ökologischer Faktor. Schweiz. Z. Hydrol. 21/2, 133264 (1959).

2 Axt, G.: Ein Gerät zur kontinuierlichen Messung des organischen Kohlenstoffs in Trinkwasser- und Abwasserbereichen. Haus Tech., Vortragsveröff. 231, 21-28 (1970).

3 Bick, H.: Ciliated Protozoa, 198 S. WHO, Genf 1972.

4 Bick, H.: Ciliaten als Indikatororganismen bei der Gewässeruntersuchung. Daten und Dokumente zum Umweltschutz, H. 19 (1976).

5 Bick, H., und Kunze, S.: Eine Zusammenstellung von autökologischen und saprobiologischen Befunden an Süsswasserciliaten. Int. Revue ges. Hydrobiol. 56/3, 337-384 (1971).

6 Buck, H.: Statistische Untersuchungen zur Saprobität und zum Leitwert verschiedener Organismen. Münchn. Beitr. Abwass.- Fisch.- Flussbiol. 19, 14-44 (1971).

7 Eidg. Amt für Wasserwirtschaft, Mitt. Nr. 33 (1939): Untersuchungen in der Natur über Bettbildung, Geschiebe- und Schwebestofführung. 
8 Heuss, K.: Untersuchung zur Bewertung von Verfahren der biologischen Gewässer-Beurteilung. Schr.-Reihe Landesanst. Wass. Abfall Nordrh.-Westf. 36, 177 S. (1976).

9 Kahl, A.: Wimpertiere oder Ciliata (Infusoria). In: Dahl, M., und Bischoff, H. (Hrsg.): Tierwelt Deutschlands, Teile 18, 21, 25, 30. Fischer, Jena 1930-1935.

10 Kolkwitz, R., und Marsson, M.: Ökologie der tierischen Saprobien. Int. Revue Ges. Hydrobiol. 2, 126-152 (1909).

11 Liebmann, H.: Handbuch der Frischwasser- und Abwasserbiologie, Bd. 1, 539 S. R. Oldenbourg, München 1951.

12. Müller, R., und Wiedemann, O.: Die Bestimmung des Nitrat-Jons im Wasser. Vom Wass. 22, 247271 (1955).

13 Nusch, E. A.: Ökologische und systematische Untersuchungen der Peritricha (Protozoa, Ciliata) im Aufwuchs von Talsperren und Flußstauen mit verschiedenem Saprobitätsgrad. Arch. Hydrobiol., Suppl. 37, 243-386 (1970).

14 Patton, J., und Reeder, W.: New Indicator for Titration of Calcium with (Ethylenedinitrilo) Tetraacete. Analyt. Chem. 28/6, 1026 S. (1956).

15 Perret, P.: Zustand der schweizerischen Fliessgewässer in den Jahren 1974/75. Eidg. Amt für Umweltschutz (1977).

16 Ruttner, F.: Grundriss der Limnologie, 332 S. Walter de Gruyter, Berlin 1962.

17 Schmerenbeck, W.: Experimentelle Untersuchungen an strömenden Modellgewässern zur Frage der Beziehung zwischen dem Abbau organischer Substanz und der Ciliatenbesiedelung. Diss. Univ. Bonn (1974).

18 Schmid, M.: Die Bestimmung kleiner Mengen von organischem Stickstoff im Wasser von Binnenseen, Schweiz. Z. Hydrol. 30/1, 244-266 (1968).

19 Schmid, M., und Ambühl, H.: Die Bestimmung geringster Mengen von Gesamtphosphor im Wasser von Binnenseen. Schweiz. Z. Hydrol. 27/1, 184-192 (1965).

20 Sládeček, V.: System of Water Quality from the Biological Point of View. Arch. Hydrobiol, Beih. 7 , 218 S. (1973).

21 Sládečková, A., und Sládeček, V.: The indicator value of some sessile protozoa. Arch. Protistenk. 109, 223-225 (1966).

22 Steinmann, P., und Surbeck, G.: Die Wirkung organischer Verunreinigungen auf die Fauna schweizerischer fliessender Gewässer, 452 S. Schweiz. Departement des Innern (1918).

23 Verordnung über Abwassereinleitungen vom 8.12.1975. Syst. Sammlung des Bundesrechtes, Ziff. 814.225.21, Bern.

24 Winkler, L. W.: Die Bestimmung des im Wasser gelösten Sauerstoffs. Ber. dt. chem. Ges. 21, 28432854 (1888).

25 Zelinka, M., und Marvan, P.: Zur Präzisierung der biologischen Klassifikation der Reinheit fliessender Gewässer. Arch. Hydrobiol. 57/3, 389-407 (1961).

26 Zobrist, J., Davis, J.S., und Hegi, H.R.: Charakterisierung des chemischen Zustandes des Flusses Glatt. Gas Wass. Abwass. 56, 97-114 (1976).

27 Zobrist, J., Davis, J.S., und Hegi, H. R.: Charakterisierung des chemischen Zustandes von Fliessgewässern. Gas Wass. Abwass. 57, 402-415 (1977).

Adresse des Autors: F. Stössel, dipl, phil. II, EAWAG, CH-8600 Dübendorf. 